\title{
Provider costs for prevention and treatment of cardiovascular and related conditions in low- and middle-income countries: a systematic review
}

\author{
Elizabeth D. Brouwer ${ }^{1 *}$, David Watkins ${ }^{2}$, Zachary Olson ${ }^{3}$, Jane Goett ${ }^{4}$, Rachel Nugent ${ }^{1}$ and Carol Levin ${ }^{1}$
}

\begin{abstract}
Background: The burden of cardiovascular disease (CVD) and CVD risk conditions is rapidly increasing in low- and middle-income countries, where health systems are generally ill-equipped to manage chronic disease. Policy makers need an understanding of the magnitude and drivers of the costs of cardiovascular disease related conditions to make decisions on how to allocate limited health resources.

Methods: We undertook a systematic review of the published literature on provider-incurred costs of treatment for cardiovascular diseases and risk conditions in low- and middle-income countries. Total costs of treatment were inflated to 2012 US dollars for comparability across geographic settings and time periods.

Results: This systematic review identified 60 articles and 143 unit costs for the following conditions: ischemic heart disease, non-ischemic heart diseases, stroke, heart failure, hypertension, diabetes, and chronic kidney disease. Cost data were most readily available in middle-income countries, especially China, India, Brazil, and South Africa. The most common conditions with cost studies were acute ischemic heart disease, type 2 diabetes mellitus, stroke, and hypertension.

Conclusions: Emerging economies are currently providing a base of cost evidence for NCD treatment that may prove useful to policy-makers in low-income countries. Initial steps to publicly finance disease interventions should take account of costs. The gaps and limitations in the current literature include a lack of standardized reporting as well as sparse evidence from low-income countries.
\end{abstract}

Keywords: Cardiovascular disease, CVD, Systematic review, Economic evaluation, Non-communicable disease, Cost analysis

\section{Background}

Cardiovascular disease (CVD) and CVD risk conditions, such as hypertension, type 2 diabetes mellitus (T2DM), and chronic kidney disease (CKD), are the leading cause of global morbidity and mortality [1]. Increasingly larger proportions of the global population are exposed to risk of non-communicable disease (NCD), such as urbanization, tobacco use, and sedentary behavior [2]. The growing

\footnotetext{
* Correspondence: ebrouwer@uw.edu

'Disease Control Priorities Network, Department of Global Health, University of Washington, 325 Ninth Avenue, Box 259931, Seattle, WA 98104, USA
} Full list of author information is available at the end of the article prevalence of these conditions is particularly troublesome in low- and middle-income countries (LMICs), where agespecific rates for NCD mortality are nearly twice as high as in high-income countries [3], and treatment can be very costly [4]. There is evidence that health systems in these settings are already strained by the dual burden of infectious and chronic disease, especially in resource-limited settings [5]. Many people suffering from CVD in LMICs remain untreated, or their conditions are poorly managed, due to lack of access to primary health care and high costs [6].

Efforts to improve prevention and management of NCDs in LMICs are increasing. Mortality reduction 
targets, such as the World Heart Federation's $25 \times 25$ and the Lancet's $40 \times 30$, are unprecedented in putting NCDs on the international agenda [7, 8]. The resources required in order to meet these targets in any given LMIC are less clear. Economic evaluations are especially important for settings that do not yet have policies or the infrastructure in place to address these emerging issues [9]. Unfortunately, financial and economic cost estimates of these interventions in LMICs are limited. In order to raise awareness of the economic costs of CVD and to support increased allocation of resources for addressing CVD and risk conditions, accurate cost data are needed by donors, ministries of health and economic modelers.

Recent reviews of household burden or costeffectiveness of CVD interventions in LMICs have been published $[6,10-13]$; however, there are no recent reviews, to our knowledge, of provider-incurred costs of interventions for CVD and risk conditions. We therefore reviewed literature on the providerincurred costs for the treatment of cardiovascular and related risk conditions in health system settings of LMICs. Specifically, we consider diabetes, chronic kidney disease, hypertension and hyperlipidemia, stroke, ischemic heart disease, and non-ischemic heart diseases. We chose these conditions because of their interrelated risk factors and the large overlap in clinical resources that are required to management them. We will refer to the entire grouping as "CVRD" (cardiovascular and related diseases) throughout the remainder of the paper.

Our primary objective was to present the level and variability in direct medical or programmatic costs for treating CVRD in LMICs. Our secondary objective was to identify gaps in knowledge and suggest future economic research needs to support policy development, resource allocation, and decision-making.

\section{Methods}

\section{Search strategy and article retrieval}

We conducted our review according to the PRISMA guidelines [14]. We searched the following databases: Medline, EMBASE, NHS-EED, HEED and EconLit. We adapted the search terms specifically to each database, using 1) terms related to cardiovascular diseases and risk conditions plus 2) terms for LMICs and regions plus 3) terms denoting economic evaluation. Additional file 1 details the full strategy. We limited our results to articles published on or after January $1^{\text {st }}, 2000$ until approximately July $1^{\text {st }}, 2014$. We screened eligible titles and abstracts to determine which articles would be included for final review. Two independent reviewers reviewed the full text articles. In addition, we examined the references of the included articles and prior reviews for potentially relevant studies. Broadly, the following criteria were used to determine inclusion:

- Included an economic evaluation

- Included evaluation of at least one low- or middleincome country (as defined by the World Bank)

- Referred to our previously standardized list of cardiovascular or related-related conditions

- Made available in English

- Published on or after January 2000.

We used both the Drummond Checklist and Mogyorosy and Smith's 2012 literature review for guidance in creating the following guidelines regarding quality and inclusion $[15,16]$ :

- The study must have conducted at least one type of economic evaluation, including cost analysis, costeffective analysis, or cost-utility analysis, with clearly presented unit cost data.

- We did not consider costs with health outcomes as a denominator, such as disability-adjusted life years (DALYs), quality adjusted life years (QALYs), or lifeyears saved (LYS).

- The study must have utilized either original unit cost data regarding a CVRD intervention delivered in a health systems setting, or unit costs from a credible and known source, such as WHOCHOICE. We did not consider population-level interventions or policy costs.

- The study must have presented direct intervention costs (medical or programmatic) from the provider perspective, regardless of payer or indirect costs. Studies that considered multiple perspectives were only considered if they clearly delineated provider costs. Provider costs were defined as those delivering the services, such as those incurred by health systems, hospitals or clinics, governmental bodies, funders, or programs.

- The study had to meet certain quality standards, which includes having a description of the intervention and analysis, detailing the time and location of data collection, and clearly stating the year and currency of presented costs.

To inform the organization and synthesis of the literature across conditions and interventions, we created a conceptual framework of the clinical and public health considerations in CVD treatment (Fig. 1). There are several points at which one can intervene on cardiovascular risk factors, risk conditions, and CVDs themselves, both within and outside of the health system. This review examines only the costs that exist within the health sector, and health facilities 


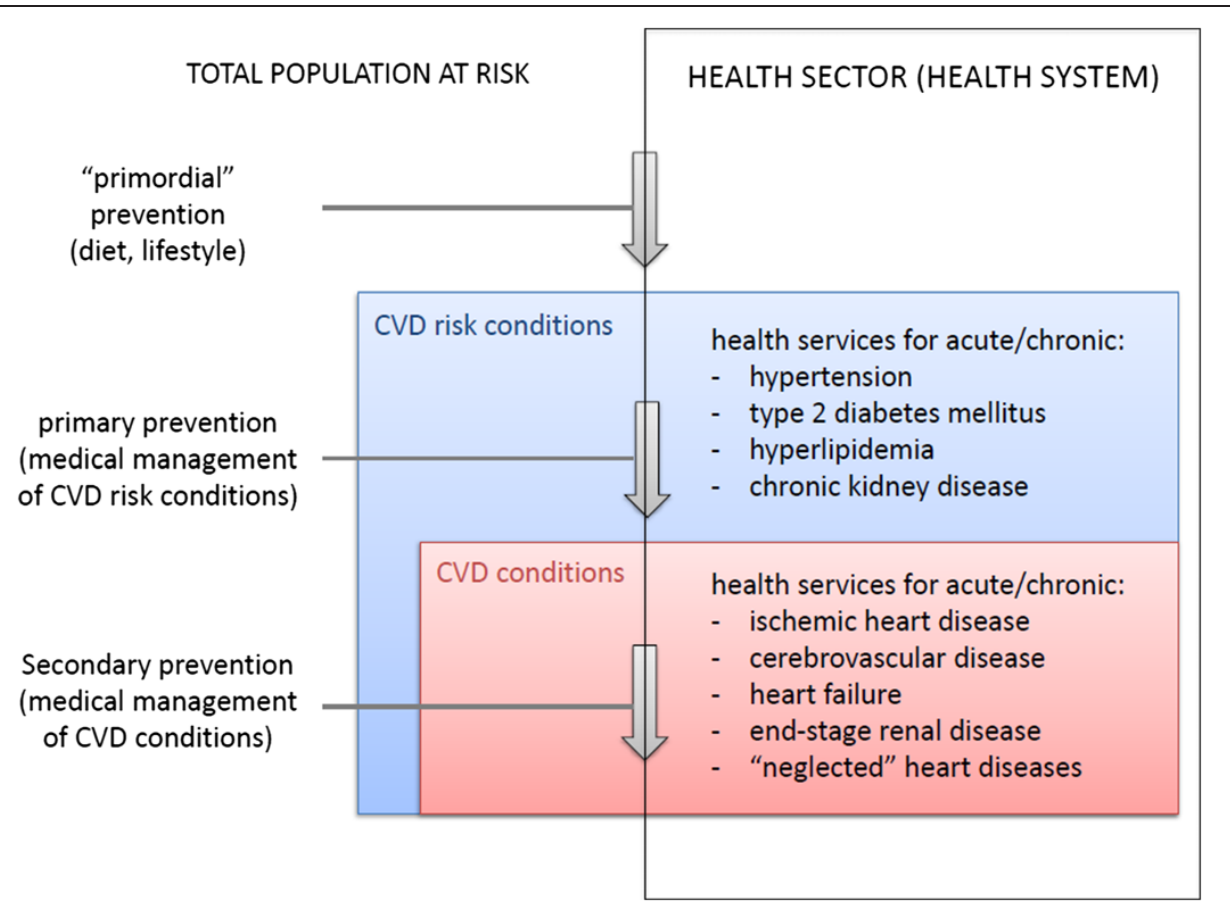

Fig. 1 CVRD Conceptual Framework. This framework informed our inclusion criteria and subsequent data extraction and organization

specifically, but across the spectrum of cardiovascular risk conditions and diseases themselves.

\section{Data extraction}

For each eligible study we entered the following information into an electronic database: condition(s), country or region, target population, type of treatment or intervention, and level of care. We also noted study methods, such as sample size and strategy, exchange rate, discount rate, sensitivity analysis, and cost categories. The total treatment or intervention costs, defined as the total cost per patient, were extracted in their original currency and year. For acute events, we used the total cost per patient as presented. For recurring costs, such as hemodialysis treatments or ongoing hypertension management, we calculated the cost per treatment or per year. Differences in inpatient and outpatient costs were noted where reported and applicable. Of note, most of the included studies reported multiple costs, particularly if they discussed more than one CVRD intervention or more than one geographic setting.

We converted all costs to US Dollars (2012), the currency and year in which we present all costs in this paper. When possible, we extracted the data in local currency units (LCUs), inflated it to 2012 rates using the World Bank consumer price indices, and converted it to 2012 US Dollars via World Bank rates. When costs were presented for regions, we used the largest LMIC country in the region as a proxy. For data presented in international dollars, we converted it back into LCUs using World Bank Purchasing Power Parity, then we followed our standard processes. Once the data were in a common currency, we grouped similar interventions and qualitatively compared the magnitudes and variability of total and input costs. We combined data into the two overarching groups to reflect our aforementioned framework: management of CVD risk conditions and treatment of acute and chronic CVDs. The former group included hypertension, hyperlipidemia, CKD, T2DM, and generic CVD prevention. The latter group focused specifically on ischemic heart disease, nonischemic heart diseases, stroke, heart failure, T2DM sequelae (specifically, microvascular complications such as foot ulcers and retinopathy), and end-stage renal disease (ESRD), which is due predominately to poorly controlled hypertension and T2DM.

\section{Results}

The results of the systematic search process can be found in Fig. 2. 60 studies met our final inclusion criteria, presenting approximately 143 unit costs reported in Table 1. An additional 121 studies were reviewed at the full text stage but were excluded on the basis of our inclusion criteria or for methodological reasons, i.e., they did not meet the criteria in our quality checklist.

The number of published articles for CVD costs in LMICs has increased dramatically since 2000 , as seen in Fig. 3. In fact, we identified only five articles in the years 2000-2006, increasing to 16 articles published in 2013, 


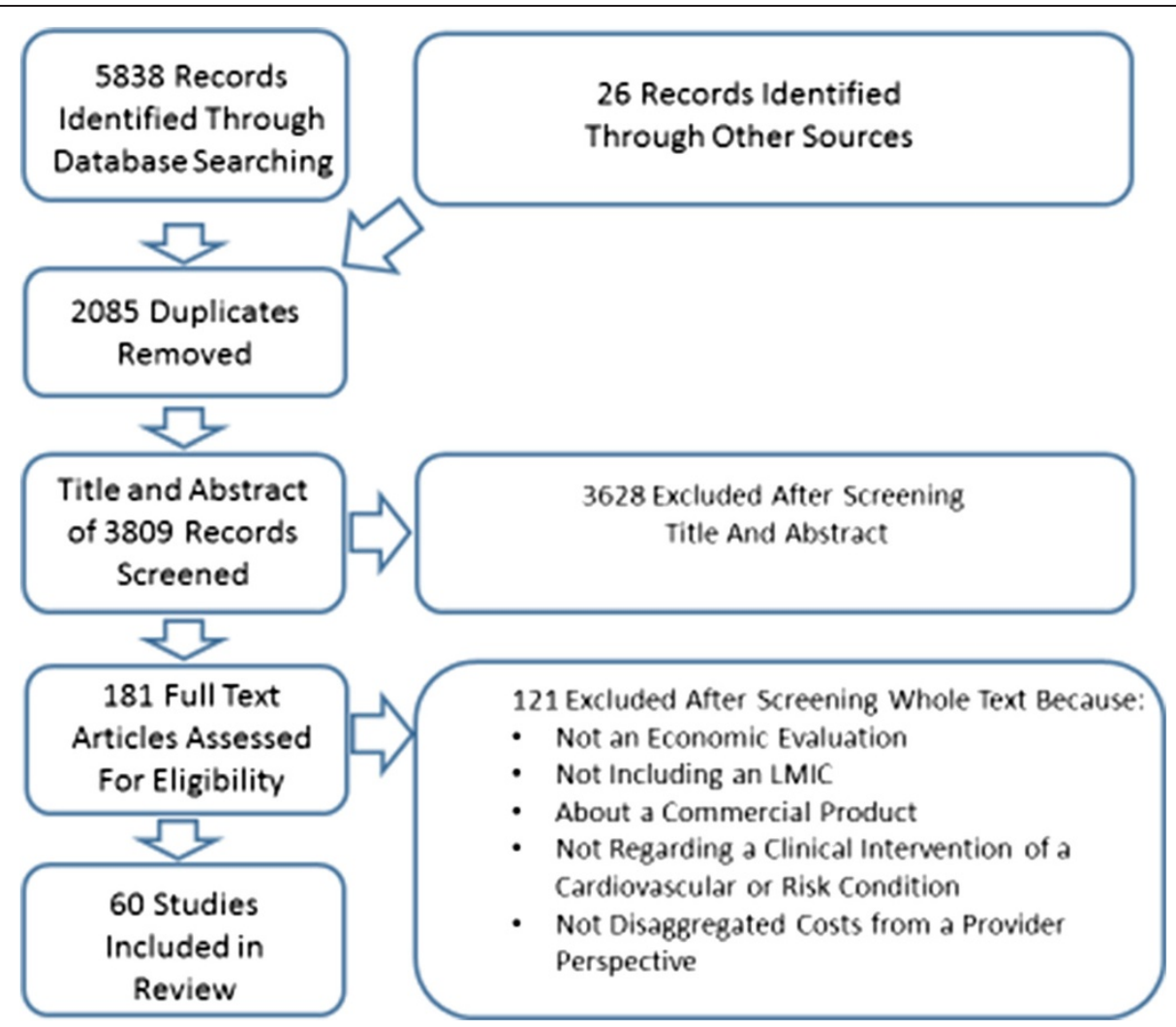

Fig. 2 Article Selection Flow Chart (PRISMA STANDARDS). Flow diagram for the selection of published articles evaluating the costs of providing preventive care or treatment for cardiovascular disease, diabetes, and chronic kidney disease in low- and middle-income countries

the last complete year of our review. Most of the cost data occurred either in urban areas or at the national level of middle income countries, with only four studies considering low-income countries. The sample is mainly from Asian countries (34 articles), dominated by studies from India (8 articles) and China (12 articles). Almost a quarter of the cost data came from China; India contributed the next highest frequency of cost data (14\%). One-third of our cost data concerned diabetes, which was a considerably higher proportion than any other condition, and two-thirds of those diabetes costs were from Asian-based studies.

We obtained cost data from a variety of economic evaluations. Almost two-thirds of the articles $(n=37)$ were cost analyses, meaning they only collected and presented data on costs, not health outcomes. Thirty studies determined the total costs of interventions by reviewing hospital records while 29 studies used an ingredients approach, taking the sum cost of activities and resources used to find the total costs. Only one study used a questionnaire to collect data. Of the 60 studies, over half converted their costs in US Dollars or the hypothetical currency "International Dollars" for presentation (32 articles). Although it is difficult to measure the quality of a study objectively, economic evaluation guidelines recommend discounting future costs and conducting sensitivity analyses when assumptions are made. We noted that approximately one-third of the 60 studies conducted sensitivity analysis, and one-fourth used discounting in their methods; 11 articles used both.

\section{Management of cardiovascular risk conditions}

Our review identified 60 cost estimates from 31 articles for clinical management of CVD risk conditions such as hypertension, hyperlipidemia, chronic kidney disease and T2DM). There were also brief mentions of pharmacological tobacco cessation measures, rheumatic fever and generic CVD prevention strategies such as polypill use. More than three-quarters of this data came from Asian countries, and more than half from China, India or Thailand.

We found 14 unit costs for hypertension management in LMICs, with drug therapy ranging from \$2.21-\$76.29. Using general practitioners in community programs cost only $\$ 0.81-\$ 8.67$ per patient per year, compared to annual outpatient costs to manage hypertension, which ranged from $\$ 38.00$ to $\$ 565.54$ per patient. Only three studies mentioned the costs of managing dyslipidemia, which were similar to managing hypertension. One study looked 
Table 1 Unit cost data for the treatment of cardiovascular risk conditions (USD 2012)

\begin{tabular}{|c|c|c|c|c|c|c|c|}
\hline $\begin{array}{l}\text { Sub-Condition } \\
\text { (If Applicable) }\end{array}$ & Intervention or Treatment & Country & $\begin{array}{l}\text { Cost Presented in } \\
\text { Paper }\end{array}$ & Unit & $\begin{array}{l}\text { Currency } \\
\text { (Year) }\end{array}$ & $\begin{array}{l}\text { Cost in USD } \\
(2012)\end{array}$ & Unit \\
\hline \multicolumn{8}{|c|}{ Management of cardiovascular risk conditions } \\
\hline \multicolumn{8}{|l|}{ Hypertension } \\
\hline & Hypertension Drug Treatment [34] & Nigeria & $300.00-2,100.00$ & Per month & $\begin{array}{l}\text { Nigeria } \\
(2010)\end{array}$ & $\$ 2.38-\$ 16.66$ & $\begin{array}{l}\text { Per patient } \\
\text { per year }\end{array}$ \\
\hline & $\begin{array}{l}\text { Pharmacological high blood pressure and } \\
\text { cholesterol treatment [35] }\end{array}$ & Tanzania & $1.57-54.28$ & Per year & US (2005) & $\$ 2.21-\$ 76.29$ & $\begin{array}{l}\text { Per patient } \\
\text { per year }\end{array}$ \\
\hline & $\begin{array}{l}\text { Pharmacological high blood pressure } \\
\text { treatment [33] }\end{array}$ & Argentina & 49.72 & $\begin{array}{l}\text { Per patient } \\
\text { per year }\end{array}$ & US (2007) & $\$ 52.24$ & $\begin{array}{l}\text { Per patient } \\
\text { per year }\end{array}$ \\
\hline & $\begin{array}{l}\text { Community Hypertension Control Program: } \\
\text { Home Health Education and General } \\
\text { Practitioner [36] }\end{array}$ & Pakistan & 3.99 & $\begin{array}{l}\text { Per patient } \\
\text { per year }\end{array}$ & US (2007) & $\$ 4.96$ & $\begin{array}{l}\text { Per patient } \\
\text { per year }\end{array}$ \\
\hline & $\begin{array}{l}\text { Community Hypertension Control Program: } \\
\text { Home Health Education [36] }\end{array}$ & Pakistan & 3.34 & $\begin{array}{l}\text { Per patient } \\
\text { per year }\end{array}$ & US (2007) & $\$ 4.15$ & $\begin{array}{l}\text { Per patient } \\
\text { per year }\end{array}$ \\
\hline & $\begin{array}{l}\text { Community Hypertension Control Program: } \\
\text { General Practitioner [36] }\end{array}$ & Pakistan & 0.65 & $\begin{array}{l}\text { Per patient } \\
\text { per year }\end{array}$ & US (2007) & $\$ 0.81$ & $\begin{array}{l}\text { Per patient } \\
\text { per year }\end{array}$ \\
\hline & $\begin{array}{l}\text { Community Hypertension Reduction } \\
\text { Program (1-year) [37] }\end{array}$ & China & 7.17 & $\begin{array}{l}\text { Per patient } \\
\text { per year }\end{array}$ & US (2009) & $\$ 8.67$ & $\begin{array}{l}\text { Per patient } \\
\text { per year }\end{array}$ \\
\hline & $\begin{array}{l}\text { Guideline-Oriented Training Program for } \\
\text { Hypertension Control in Community } \\
\text { Health Centers [38] }\end{array}$ & China & 79.30 & Per year & US (2002) & $\$ 138.96$ & Per year \\
\hline & Hypertension Outpatient Treatment [32] & $\begin{array}{l}\text { South } \\
\text { Africa }\end{array}$ & 86.00 & $\begin{array}{l}\text { Per patient } \\
\text { per year }\end{array}$ & US (2001) & $\$ 169.28$ & $\begin{array}{l}\text { Per patient } \\
\text { per year }\end{array}$ \\
\hline & Hypertension Outpatient Treatment [39] & China & 487.30 & $\begin{array}{l}\text { Per patient } \\
\text { per year }\end{array}$ & US (2010) & $\$ 565.54$ & $\begin{array}{l}\text { Per patient } \\
\text { per year }\end{array}$ \\
\hline & $\begin{array}{l}\text { Hypertension Outpatient Treatment at } \\
\text { Urban Health Clinic [40] }\end{array}$ & Thailand & 916.54 & $\begin{array}{l}\text { Per patient } \\
\text { per year }\end{array}$ & $\begin{array}{l}\text { Thailand } \\
\text { (1999) }\end{array}$ & $\$ 41.42$ & $\begin{array}{l}\text { Per patient } \\
\text { per year }\end{array}$ \\
\hline & Hypertension Outpatient Treatment [41] & China & 96.90 & $\begin{array}{l}\text { Per } \\
\text { outpatient } \\
\text { visit }\end{array}$ & $\begin{array}{l}\text { China } \\
\text { (2003) }\end{array}$ & $\$ 20.28$ & $\begin{array}{l}\text { Per } \\
\text { outpatient } \\
\text { visit }\end{array}$ \\
\hline & $\begin{array}{l}\text { Hypertension Outpatient Treatment and } \\
\text { Medications [42] }\end{array}$ & Tanzania & 38.00 & $\begin{array}{l}\text { Per patient } \\
\text { per year }\end{array}$ & US (2012) & $\$ 38.00$ & $\begin{array}{l}\text { Per patient } \\
\text { per year }\end{array}$ \\
\hline & Hypertension Inpatient Visit [41] & China & $3,904.00$ & $\begin{array}{l}\text { Per } \\
\text { inpatient } \\
\text { stay }\end{array}$ & $\begin{array}{l}\text { China } \\
(2003)\end{array}$ & $\$ 817.10$ & $\begin{array}{l}\text { Per } \\
\text { inpatient } \\
\text { stay }\end{array}$ \\
\hline & $\begin{array}{l}\text { Hypertensive Emergency Treatment Cost } \\
\text { (Inpatient and Outpatient) [43] }\end{array}$ & $\begin{array}{l}\text { Congo, } \\
\text { Rep. }\end{array}$ & $159,600.00$ & Per patient & $\begin{array}{l}\text { Congo, Rep. } \\
\text { (2006) }\end{array}$ & $\$ 400.90$ & Per patient \\
\hline \multicolumn{8}{|l|}{ Hyperlipidemia } \\
\hline & $\begin{array}{l}\text { Screening for dyslipidemia among healthy } \\
35-39 \text { year olds [44] }\end{array}$ & Thailand & 127.22 & $\begin{array}{l}\text { Per patient } \\
\text { screened }\end{array}$ & $\begin{array}{l}\text { Thailand } \\
\text { (2008) }\end{array}$ & $\$ 4.48$ & $\begin{array}{l}\text { Per patient } \\
\text { screened }\end{array}$ \\
\hline & $\begin{array}{l}\text { Screening for dyslipidemia among healthy } \\
35-60 \text { year olds [45] }\end{array}$ & Thailand & $1,043.60$ & $\begin{array}{l}\text { Per case } \\
\text { detected }\end{array}$ & $\begin{array}{l}\text { Thailand } \\
\text { (2008) }\end{array}$ & $\$ 36.77$ & $\begin{array}{l}\text { Per case } \\
\text { detected }\end{array}$ \\
\hline & $\begin{array}{l}\text { Pharmacological high cholesterol } \\
\text { treatment [33] }\end{array}$ & Argentina & 118.79 & $\begin{array}{l}\text { Per patient } \\
\text { per year }\end{array}$ & US (2007) & $\$ 124.81$ & $\begin{array}{l}\text { Per patient } \\
\text { per year }\end{array}$ \\
\hline & Home visit by Health Care Professional [40] & Thailand & 574.86 & Per visit & $\begin{array}{l}\text { Thailand } \\
\text { (1999) }\end{array}$ & $\$ 25.98$ & Per visit \\
\hline \multicolumn{8}{|c|}{ Chronic Kidney Disease } \\
\hline & $\begin{array}{l}\text { Amlodipine (Anti-hypertensive Drug } \\
\text { Therapy for Patients with Diabetes, } \\
\text { Hypertension, and Nephropathy) [29] }\end{array}$ & China & $2,013.00$ & Per year & US (2004) & $\$ 3,356.78$ & $\begin{array}{l}\text { Per patient } \\
\text { per year }\end{array}$ \\
\hline & $\begin{array}{l}\text { Irbesartan (Anti-hypertensive Drug Therapy } \\
\text { for Patients with Diabetes, Hypertension, } \\
\text { and Nephropathy) [29] }\end{array}$ & China & $1,660.00$ & Per year & US (2004) & $\$ 2,768.13$ & $\begin{array}{l}\text { Per patient } \\
\text { per year }\end{array}$ \\
\hline & & Malaysia & 332.00 & Per year & US (2004) & $\$ 503.07$ & \\
\hline
\end{tabular}


Table 1 Unit cost data for the treatment of cardiovascular risk conditions (USD 2012) (Continued)

Amlodipine (Anti-hypertensive Drug Therapy for Patients with Diabetes, Hypertension, and Nephropathy) [29]

Irbesartan (Anti-hypertensive Drug Therapy for Patients with Diabetes, Hypertension, and Nephropathy) [29]

Amlodipine (Anti-hypertensive Drug Therapy for Patients with Diabetes, Hypertension, and Nephropathy) [29]

Irbesartan (Anti-hypertensive Drug Therapy for Patients with Diabetes, Hypertension, and Nephropathy) [29]

Type 2 Diabetes Mellitus

\begin{tabular}{|c|c|c|c|c|c|c|}
\hline $\begin{array}{l}\text { Screening using fasting capillary blood } \\
\text { glucose [46] }\end{array}$ & China & 10.00 & Per test & $\begin{array}{l}\text { China } \\
\text { (2009) }\end{array}$ & $\$ 1.77$ & Per patient \\
\hline Screening using Diabetes Risk Score [46] & China & 5.00 & Per test & $\begin{array}{l}\text { China } \\
(2009)\end{array}$ & $\$ 0.89$ & Per patient \\
\hline $\begin{array}{l}\text { Screening for diabetes in a community } \\
\text { health clinic [47] }\end{array}$ & China & 3.00 & Per patient & US (2007) & $\$ 4.25$ & Per patient \\
\hline Tertiary Hospital Diagnostic Test [46] & China & 95.00 & Per test & $\begin{array}{l}\text { China } \\
(2009)\end{array}$ & $\$ 16.82$ & Per patient \\
\hline $\begin{array}{l}\text { Primary Care - Behavior management } \\
\text { program to prevent diabetes [48] }\end{array}$ & India & 54.67 & $\begin{array}{l}\text { Per patient } \\
\text { per year }\end{array}$ & US (2006) & $\$ 78.93$ & $\begin{array}{l}\text { Per patient } \\
\text { per year }\end{array}$ \\
\hline $\begin{array}{l}\text { Primary Care - Metformin regimen to } \\
\text { prevent diabetes [48] }\end{array}$ & India & 53.00 & $\begin{array}{l}\text { Per patient } \\
\text { per year }\end{array}$ & US (2006) & $\$ 76.53$ & $\begin{array}{l}\text { Per patient } \\
\text { per year }\end{array}$ \\
\hline $\begin{array}{l}\text { Primary Care - Behavior management } \\
\text { program with Metformin regime to } \\
\text { prevent diabetes [48] }\end{array}$ & India & 69.67 & $\begin{array}{l}\text { Per patient } \\
\text { per year }\end{array}$ & US (2006) & $\$ 100.59$ & $\begin{array}{l}\text { Per patient } \\
\text { per year }\end{array}$ \\
\hline $\begin{array}{l}\text { Mean total annual direct medical cost } \\
\text { (without complication) [49] }\end{array}$ & China & $5,313.20$ & Per year & $\begin{array}{l}\text { China } \\
(2007)\end{array}$ & $\$ 989.16$ & $\begin{array}{l}\text { Per patient } \\
\text { per year }\end{array}$ \\
\hline $\begin{array}{l}\text { Mean total annual direct medical cost } \\
\text { (with complication) [49] }\end{array}$ & China & $13,320.10$ & Per year & $\begin{array}{l}\text { China } \\
(2007)\end{array}$ & $\$ 2,479.82$ & $\begin{array}{l}\text { Per patient } \\
\text { per year }\end{array}$ \\
\hline $\begin{array}{l}\text { Annual treatment costs (inpatient and } \\
\text { outpatient costs) [50] }\end{array}$ & Thailand & $6,331.00$ & $\begin{array}{l}\text { Per patient } \\
\text { per year }\end{array}$ & $\begin{array}{l}\text { Thailand } \\
\text { (2001) }\end{array}$ & $\$ 277.14$ & $\begin{array}{l}\text { Per patient } \\
\text { per year }\end{array}$ \\
\hline $\begin{array}{l}\text { Annual treatment costs (inpatient and } \\
\text { outpatient costs) [51] }\end{array}$ & Nigeria & $47,924.36$ & $\begin{array}{l}\text { Per patient } \\
\text { per year }\end{array}$ & $\begin{array}{l}\text { Nigeria } \\
(2010)\end{array}$ & $\$ 380.14$ & $\begin{array}{l}\text { Per patient } \\
\text { per year }\end{array}$ \\
\hline $\begin{array}{l}\text { Annual Diabetes Treatment in Community } \\
\text { Clinic (Including Inpatient and } \\
\text { Outpatient Visits) [52] }\end{array}$ & Brazil & $1,319.15$ & Per year & US (2010) & $\$ 1,335.52$ & $\begin{array}{l}\text { Per patient } \\
\text { per year }\end{array}$ \\
\hline $\begin{array}{l}\text { Annual treatment costs (inpatient and } \\
\text { outpatient costs) [53] }\end{array}$ & India & $3,006.00$ & $\begin{array}{l}\text { Per patient } \\
\text { per year }\end{array}$ & India (2012) & $\$ 56.25$ & $\begin{array}{l}\text { Per patient } \\
\text { per year }\end{array}$ \\
\hline $\begin{array}{l}\text { Urban Health Center providing primary } \\
\text { community care [40] }\end{array}$ & Thailand & $1,408.59$ & $\begin{array}{l}\text { Per patient } \\
\text { per year }\end{array}$ & $\begin{array}{l}\text { Thailand } \\
\text { (1999) }\end{array}$ & $\$ 63.66$ & $\begin{array}{l}\text { Per } \\
\text { outpatient } \\
\text { visit }\end{array}$ \\
\hline $\begin{array}{l}\text { Inpatient stay w/o hypertension } \\
\text { (Average } 7 \text { Days LOS) [54] }\end{array}$ & India & $18,650.00$ & $\begin{array}{l}\text { Per } \\
\text { inpatient } \\
\text { stay }\end{array}$ & India (2007) & $\$ 558.77$ & $\begin{array}{l}\text { Per } \\
\text { inpatient } \\
\text { stay }\end{array}$ \\
\hline $\begin{array}{l}\text { Inpatient Stay w/hypertension } \\
\text { (Average } 5 \text { Days LOS) [54] }\end{array}$ & India & $21,000.00$ & $\begin{array}{l}\text { Per } \\
\text { inpatient } \\
\text { stay }\end{array}$ & India (2007) & $\$ 629.18$ & $\begin{array}{l}\text { Per } \\
\text { inpatient } \\
\text { stay }\end{array}$ \\
\hline $\begin{array}{l}\text { Inpatient stay w/o hypertension } \\
\text { (Average } 7 \text { Days LOS) [54] }\end{array}$ & India & $28,000.00$ & $\begin{array}{l}\text { Per two } \\
\text { years }\end{array}$ & India (2007) & $\$ 419.45$ & $\begin{array}{l}\text { Per patient } \\
\text { per year }\end{array}$ \\
\hline $\begin{array}{l}\text { Inpatient Stay w/hypertension } \\
\text { (Average } 5 \text { Days LOS) [54] }\end{array}$ & India & $38,000.00$ & $\begin{array}{l}\text { Per two } \\
\text { years }\end{array}$ & India (2007) & $\$ 569.25$ & $\begin{array}{l}\text { Per patient } \\
\text { per year }\end{array}$ \\
\hline $\begin{array}{l}\text { Inpatient Treatment: without complication } \\
\text { (Average } 9.8 \text { Days LOS) [55] }\end{array}$ & China & $6,903.93$ & Per patient & $\begin{array}{l}\text { China } \\
(2006)\end{array}$ & $\$ 1,346.37$ & $\begin{array}{l}\text { Per } \\
\text { inpatient } \\
\text { stay }\end{array}$ \\
\hline
\end{tabular}

\begin{tabular}{|c|c|c|c|c|c|c|}
\hline $\begin{array}{l}\text { Screening using fasting capillary blood } \\
\text { glucose [46] }\end{array}$ & China & 10.00 & Per test & $\begin{array}{l}\text { China } \\
(2009)\end{array}$ & $\$ 1.77$ & Per patient \\
\hline Screening using Diabetes Risk Score [46] & China & 5.00 & Per test & $\begin{array}{l}\text { China } \\
(2009)\end{array}$ & $\$ 0.89$ & Per patient \\
\hline $\begin{array}{l}\text { Screening for diabetes in a community } \\
\text { health clinic [47] }\end{array}$ & China & 3.00 & Per patient & US (2007) & $\$ 4.25$ & Per patient \\
\hline Tertiary Hospital Diagnostic Test [46] & China & 95.00 & Per test & $\begin{array}{l}\text { China } \\
(2009)\end{array}$ & $\$ 16.82$ & Per patient \\
\hline $\begin{array}{l}\text { Primary Care - Behavior management } \\
\text { program to prevent diabetes [48] }\end{array}$ & India & 54.67 & $\begin{array}{l}\text { Per patient } \\
\text { per year }\end{array}$ & US (2006) & $\$ 78.93$ & $\begin{array}{l}\text { Per patient } \\
\text { per year }\end{array}$ \\
\hline $\begin{array}{l}\text { Primary Care - Metformin regimen to } \\
\text { prevent diabetes [48] }\end{array}$ & India & 53.00 & $\begin{array}{l}\text { Per patient } \\
\text { per year }\end{array}$ & US (2006) & $\$ 76.53$ & $\begin{array}{l}\text { Per patient } \\
\text { per year }\end{array}$ \\
\hline $\begin{array}{l}\text { Primary Care - Behavior management } \\
\text { program with Metformin regime to } \\
\text { prevent diabetes [48] }\end{array}$ & India & 69.67 & $\begin{array}{l}\text { Per patient } \\
\text { per year }\end{array}$ & US (2006) & $\$ 100.59$ & $\begin{array}{l}\text { Per patient } \\
\text { per year }\end{array}$ \\
\hline $\begin{array}{l}\text { Mean total annual direct medical cost } \\
\text { (without complication) [49] }\end{array}$ & China & $5,313.20$ & Per year & $\begin{array}{l}\text { China } \\
(2007)\end{array}$ & $\$ 989.16$ & $\begin{array}{l}\text { Per patient } \\
\text { per year }\end{array}$ \\
\hline $\begin{array}{l}\text { Mean total annual direct medical cost } \\
\text { (with complication) [49] }\end{array}$ & China & $13,320.10$ & Per year & $\begin{array}{l}\text { China } \\
(2007)\end{array}$ & $\$ 2,479.82$ & $\begin{array}{l}\text { Per patient } \\
\text { per year }\end{array}$ \\
\hline $\begin{array}{l}\text { Annual treatment costs (inpatient and } \\
\text { outpatient costs) [50] }\end{array}$ & Thailand & $6,331.00$ & $\begin{array}{l}\text { Per patient } \\
\text { per year }\end{array}$ & $\begin{array}{l}\text { Thailand } \\
\text { (2001) }\end{array}$ & $\$ 277.14$ & $\begin{array}{l}\text { Per patient } \\
\text { per year }\end{array}$ \\
\hline $\begin{array}{l}\text { Annual treatment costs (inpatient and } \\
\text { outpatient costs) [51] }\end{array}$ & Nigeria & $47,924.36$ & $\begin{array}{l}\text { Per patient } \\
\text { per year }\end{array}$ & $\begin{array}{l}\text { Nigeria } \\
(2010)\end{array}$ & $\$ 380.14$ & $\begin{array}{l}\text { Per patient } \\
\text { per year }\end{array}$ \\
\hline $\begin{array}{l}\text { Annual Diabetes Treatment in Community } \\
\text { Clinic (Including Inpatient and } \\
\text { Outpatient Visits) [52] }\end{array}$ & Brazil & $1,319.15$ & Per year & US (2010) & $\$ 1,335.52$ & $\begin{array}{l}\text { Per patient } \\
\text { per year }\end{array}$ \\
\hline $\begin{array}{l}\text { Annual treatment costs (inpatient and } \\
\text { outpatient costs) [53] }\end{array}$ & India & $3,006.00$ & $\begin{array}{l}\text { Per patient } \\
\text { per year }\end{array}$ & India (2012) & $\$ 56.25$ & $\begin{array}{l}\text { Per patient } \\
\text { per year }\end{array}$ \\
\hline $\begin{array}{l}\text { Urban Health Center providing primary } \\
\text { community care [40] }\end{array}$ & Thailand & $1,408.59$ & $\begin{array}{l}\text { Per patient } \\
\text { per year }\end{array}$ & $\begin{array}{l}\text { Thailand } \\
\text { (1999) }\end{array}$ & $\$ 63.66$ & $\begin{array}{l}\text { Per } \\
\text { outpatient } \\
\text { visit }\end{array}$ \\
\hline $\begin{array}{l}\text { Inpatient stay w/o hypertension } \\
\text { (Average } 7 \text { Days LOS) [54] }\end{array}$ & India & $18,650.00$ & $\begin{array}{l}\text { Per } \\
\text { inpatient } \\
\text { stay }\end{array}$ & India (2007) & $\$ 558.77$ & $\begin{array}{l}\text { Per } \\
\text { inpatient } \\
\text { stay }\end{array}$ \\
\hline $\begin{array}{l}\text { Inpatient Stay w/hypertension } \\
\text { (Average } 5 \text { Days LOS) [54] }\end{array}$ & India & $21,000.00$ & $\begin{array}{l}\text { Per } \\
\text { inpatient } \\
\text { stay }\end{array}$ & India (2007) & $\$ 629.18$ & $\begin{array}{l}\text { Per } \\
\text { inpatient } \\
\text { stay }\end{array}$ \\
\hline $\begin{array}{l}\text { Inpatient stay w/o hypertension } \\
\text { (Average } 7 \text { Days LOS) [54] }\end{array}$ & India & $28,000.00$ & $\begin{array}{l}\text { Per two } \\
\text { years }\end{array}$ & India (2007) & $\$ 419.45$ & $\begin{array}{l}\text { Per patient } \\
\text { per year }\end{array}$ \\
\hline $\begin{array}{l}\text { Inpatient Stay w/hypertension } \\
\text { (Average } 5 \text { Days LOS) [54] }\end{array}$ & India & $38,000.00$ & $\begin{array}{l}\text { Per two } \\
\text { years }\end{array}$ & India (2007) & $\$ 569.25$ & $\begin{array}{l}\text { Per patient } \\
\text { per year }\end{array}$ \\
\hline $\begin{array}{l}\text { Inpatient Treatment: without complication } \\
\text { (Average } 9.8 \text { Days LOS) [55] }\end{array}$ & China & $6,903.93$ & Per patient & $\begin{array}{l}\text { China } \\
(2006)\end{array}$ & $\$ 1,346.37$ & $\begin{array}{l}\text { Per } \\
\text { inpatient } \\
\text { stay }\end{array}$ \\
\hline
\end{tabular}

Per patient per year

Malaysia 258.00

Per year US (2004) \$390.94 Per patient per year

Thailand $\quad 779.00$

Per year

US (2004)

$\$ 1,302.06$

Per patient per year

Thailand $\quad 1,340.00$

Per year

US (2004)

$\$ 2,239.75$

Per patient per year 
Table 1 Unit cost data for the treatment of cardiovascular risk conditions (USD 2012) (Continued)

\begin{tabular}{|c|c|c|c|c|c|c|c|}
\hline & $\begin{array}{l}\text { Inpatient Treatment: with chronic } \\
\text { complications (Average 9.8 Days LOS) [55] }\end{array}$ & China & $7,193.15$ & Per patient & $\begin{array}{l}\text { China } \\
(2006)\end{array}$ & $\$ 1,402.77$ & $\begin{array}{l}\text { Per } \\
\text { inpatient } \\
\text { stay }\end{array}$ \\
\hline & Inpatient/Hospital Admission [56] & Thailand & 95.99 & Per day & US (2008) & $\$ 112.65$ & Per day \\
\hline & $\begin{array}{l}\text { Inpatient Treatment in Community Clinic } \\
\text { (No LOS Stated) [52] }\end{array}$ & Brazil & 26.32 & Per year & US (2010) & $\$ 26.65$ & $\begin{array}{l}\text { Per patient } \\
\text { per year }\end{array}$ \\
\hline & Outpatient Care [56] & Thailand & 3.94 & $\begin{array}{l}\text { Per } \\
\text { outpatient } \\
\text { visit }\end{array}$ & US (2008) & $\$ 4.63$ & $\begin{array}{l}\text { Per } \\
\text { outpatient } \\
\text { visit }\end{array}$ \\
\hline & Outpatient Care in Community Clinic [52] & Brazil & $1,216.33$ & Per year & US (2010) & $\$ 1,231.43$ & $\begin{array}{l}\text { Per patient } \\
\text { per year }\end{array}$ \\
\hline & $\begin{array}{l}\text { Outpatient treatment for patient without } \\
\text { complications [57] }\end{array}$ & India & $4,493.00$ & Per year & India (2009) & $\$ 112.05$ & $\begin{array}{l}\text { Per } \\
\text { inpatient } \\
\text { stay }\end{array}$ \\
\hline & Outpatient Treatment [58] & Nepal & 16.95 & $\begin{array}{l}\text { Per } \\
\text { outpatient } \\
\text { visit }\end{array}$ & US (2010) & $\$ 17.45$ & $\begin{array}{l}\text { Per } \\
\text { outpatient } \\
\text { visit }\end{array}$ \\
\hline & Outpatient Treatment [58] & Nepal & 130.52 & Per year & US (2010) & $\$ 134.38$ & $\begin{array}{l}\text { Per patient } \\
\text { per year }\end{array}$ \\
\hline & Outpatient Treatment [59] & Brazil & $1,014.00$ & $\begin{array}{l}\text { Per patient } \\
\text { per year }\end{array}$ & US (2007) & $\$ 1,322.64$ & $\begin{array}{l}\text { Per patient } \\
\text { per year }\end{array}$ \\
\hline & Outpatient Treatment [60] & Pakistan & $1,468.90$ & $\begin{array}{l}\text { Per } \\
\text { outpatient } \\
\text { visit }\end{array}$ & $\begin{array}{l}\text { Pakistan } \\
(2006)\end{array}$ & $\$ 32.34$ & $\begin{array}{l}\text { Per } \\
\text { outpatient } \\
\text { visit }\end{array}$ \\
\hline & Outpatient Treatment [41] & China & 163.80 & $\begin{array}{l}\text { Per } \\
\text { outpatient } \\
\text { visit }\end{array}$ & $\begin{array}{l}\text { China } \\
(2003)\end{array}$ & $\$ 34.28$ & $\begin{array}{l}\text { Per } \\
\text { outpatient } \\
\text { visit }\end{array}$ \\
\hline \multicolumn{8}{|c|}{ Other clinical management of CVD risk conditions } \\
\hline \multirow{3}{*}{$\begin{array}{l}\text { Generic } \\
\text { primary } \\
\text { prevention }\end{array}$} & Modified poly-pill strategy [33] & Argentina & 103.46 & $\begin{array}{l}\text { Per patient } \\
\text { per year }\end{array}$ & US (2007) & $\$ 108.70$ & $\begin{array}{l}\text { Per patient } \\
\text { per year }\end{array}$ \\
\hline & General Outpatient Visit [40] & Thailand & 67.82 & $\begin{array}{l}\text { Per } \\
\text { outpatient } \\
\text { visit }\end{array}$ & $\begin{array}{l}\text { Thailand } \\
\text { (1999) }\end{array}$ & $\$ 3.07$ & $\begin{array}{l}\text { Per } \\
\text { outpatient } \\
\text { visit }\end{array}$ \\
\hline & $\begin{array}{l}\text { Bupropion treatment HCW counseling } \\
\text { for tobacco cessation [33] }\end{array}$ & Argentina & 117.15 & $\begin{array}{l}\text { Per patient } \\
\text { per year }\end{array}$ & US (2007) & $\$ 123.09$ & $\begin{array}{l}\text { Per patient } \\
\text { per year }\end{array}$ \\
\hline \multirow[t]{4}{*}{$\begin{array}{l}\text { Rheumatic } \\
\text { Fever }\end{array}$} & $\begin{array}{l}\text { Prevention of Rheumatic Fever (throat } \\
\text { culture) }\end{array}$ & India & $1,088.56$ & Per patient & India (2007) & $\$ 32.61$ & Per patient \\
\hline & Post rheumatic fever prophylaxis [61] & India & 879.35 & Per patient & India (2007) & $\$ 26.35$ & Per patient \\
\hline & $\begin{array}{l}\text { Inpatient Treatment for acute rheumatic } \\
\text { fever [62] }\end{array}$ & $\begin{array}{l}\text { South } \\
\text { Africa }\end{array}$ & $2,958.00$ & Per patient & US (2010) & $\$ 2,927.41$ & Per patient \\
\hline & $\begin{array}{l}\text { Echocardiographic screening for rheumatic } \\
\text { heart disease in schoolchildren [63] }\end{array}$ & Fiji & 2.07 & $\begin{array}{l}\text { Per patient } \\
\text { screened }\end{array}$ & US (2008) & $\$ 2.27$ & $\begin{array}{l}\text { Per patient } \\
\text { screened }\end{array}$ \\
\hline
\end{tabular}

at the costs of managing chronic kidney disease across China, Malaysia and Thailand, with China being more expensive than Malaysia by a scale of six to eight. We identified 30 unit costs for managing diabetes. Two studies produced four costs on diagnosing diabetes, the most expensive of which were based in tertiary hospitals. Seven studies presented ten annual per patient costs of managing diabetes in a clinical setting, which ranged from $\$ 56.25$ in India to $\$ 2,479.82$ in China; the costs were higher in cases with complications and sequelae. Nine studies also presented 16 costs on inpatient and outpatient treatment for diabetes. Inpatient stays per bed-day ranged from \$59.92-\$143.14 with an average length of stay of around 7 days. An outpatient visit ranged from $\$ 4.63-\$ 34.28$, and we also found a ten-fold variation in yearly outpatient costs.

\section{Treatment and management of acute and chronic cardiovascular conditions}

Our review also identified 83 cost estimates from 34 studies regarding the treatment of ischemic heart disease 


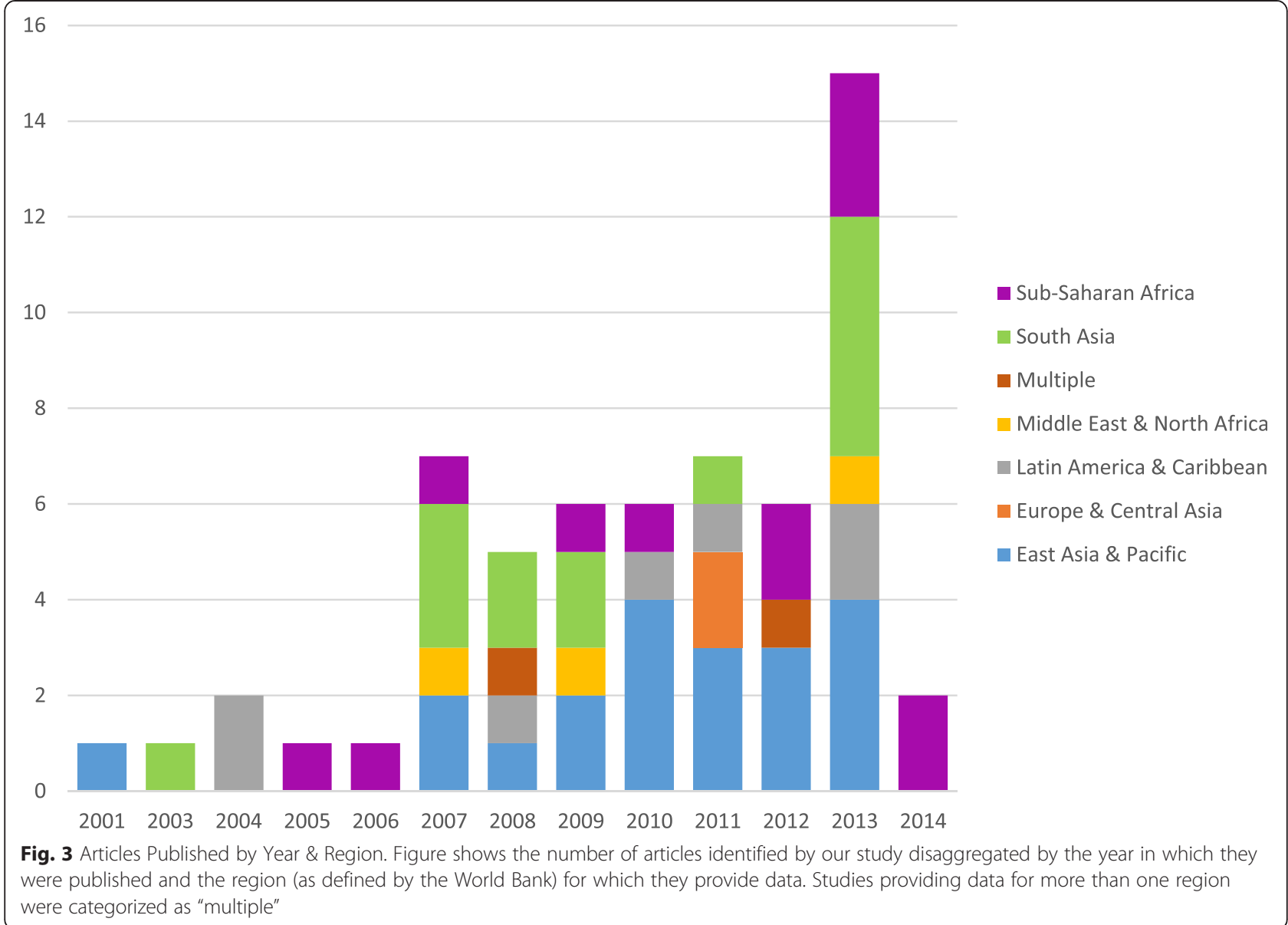

(IHD), stroke, heart failure, non-ischemic heart diseases, T2DM sequelae, and end-stage renal disease (ESRD).

Treatment of IHD typically consists of diagnostic procedures, surgical or other advanced medical procedures, and post-operative and long-term outpatient care. We found 8 articles analyzing IHD treatment with a total of 18 unit costs. An inpatient visit averaged $\$ 8,800$, with a range from $\$ 455$ to $\$ 22,500$. The highest inpatient costs were associated with longer hospitalization periods, more severe conditions, and surgical interventions. The costs of surgical procedures, including catheter-based procedures (stenting and angioplasty) or coronary artery bypass surgery (CABG), ranged from \$4,000-\$22,000 per patient per procedure depending on the diagnostics, the complexity of the procedure, and whether medical therapy and recovery costs were included in the total cost estimates.

Our search returned 15 articles and 24 unit costs for both ischemic stroke (IS) and hemorrhagic stroke (HS), though many studies did not specify the type of stroke. In most settings, IS is the more common of the two, is generally easier to treat, and has a lower case-fatality rate. Four articles compared the costs $\mathrm{HS}$ and IS treatment [17-20]. Hemorrhagic strokes were generally more severe, required longer hospital stays, and involved surgical or intensive care unit costs; HS treatment costs were between $0 \%$ and $50 \%$ higher than IS treatment. The majority of articles provided the inpatient cost per stroke event, but did not distinguish the type of strokes. The average inpatient cost of a stroke was $\$ 3,240$, but this varied widely by geographic setting, technical resources used, and average length of stay.

Five studies reported costs of T2DM sequelae, namely, diabetic foot ulcers and retinopathy. Severity of these impairments and costs saved by screening drove the total costs of both, though in opposite directions [21-25]. For instance, screening for retinopathy was generally below $\$ 30$, while diabetic foot ulcers ranged from less than $\$ 1$ to over $\$ 7,000$ in the cases of infection and amputation.

Five studies reported costs of end-stage renal disease (ESRD), primarily long-term dialysis or renal transplantation. The cost of one session of hemodialysis for ESRD ranged $\$ 79$ to $\$ 97$ [26-28], while one study estimated average annual dialysis costs at $\$ 61,000$ in Southeast Asia [29]. Kidney transplantation and post-operative care 
Table 2 Unit cost data for the treatment of cardiovascular disease and related condition (USD 2012)

\begin{tabular}{|c|c|c|c|c|c|c|c|}
\hline \multicolumn{8}{|c|}{ Treatment and Management of Acute/Chronic Cardiovascular and Related Conditions } \\
\hline \multicolumn{8}{|c|}{ Ischemic Heart Disease } \\
\hline & Outpatient Visit (All direct medical costs) [41] & China & 245.40 & $\begin{array}{l}\text { Per } \\
\text { outpatient } \\
\text { visit }\end{array}$ & $\begin{array}{l}\text { China } \\
\text { (2003) }\end{array}$ & $\$ 51.36$ & $\begin{array}{l}\text { Per } \\
\text { outpatient } \\
\text { visit }\end{array}$ \\
\hline & $\begin{array}{l}\text { CABG Procedure (Procedure and Medical } \\
\text { Therapy) [32] }\end{array}$ & $\begin{array}{l}\text { South } \\
\text { Africa }\end{array}$ & $11,431.00$ & Per patient & $\begin{array}{l}\text { US } \\
(2001)\end{array}$ & $\$ 22,500.46$ & Per patient \\
\hline & $\begin{array}{l}\text { CABG Procedure (Procedure only, no } \\
\text { medical therapy) [64] }\end{array}$ & China & $7,300.00$ & Per patient & $\begin{array}{l}\text { US } \\
(2007)\end{array}$ & $\$ 10,339.01$ & Per patient \\
\hline & $\begin{array}{l}\text { CABG Procedure (Procedure and Medical } \\
\text { Therapy) [65] }\end{array}$ & Armenia & $3,368.19$ & Per patient & $\begin{array}{l}\text { US } \\
\text { (2005) }\end{array}$ & $\$ 5,546.46$ & Per patient \\
\hline & $\begin{array}{l}\text { CABG Procedure (Procedure and Medical } \\
\text { Therapy) [66] }\end{array}$ & India & $8,055.00$ & Per patient & $\begin{array}{l}\text { US } \\
(2006)\end{array}$ & $\$ 11,630.62$ & Per patient \\
\hline & $\begin{array}{l}\text { Stenting Procedure (Procedure only, no } \\
\text { medical therapy) [64] }\end{array}$ & China & $10,000.00$ & Per patient & $\begin{array}{l}\text { US } \\
(2007)\end{array}$ & $\$ 14,163.03$ & Per patient \\
\hline & $\begin{array}{l}\text { Catheter-based revascularization Inpatient } \\
\text { Treatment [32] }\end{array}$ & $\begin{array}{l}\text { South } \\
\text { Africa }\end{array}$ & $4,737.00$ & Per patient & $\begin{array}{l}\text { US } \\
(2001)\end{array}$ & $\$ 9,324.18$ & Per patient \\
\hline & $\begin{array}{l}\text { Total operative procedures and examinations } \\
\text { [67] }\end{array}$ & Brazil & 444.29 & Per patient & $\begin{array}{l}\text { Brazil } \\
(2001)\end{array}$ & $\$ 455.47$ & Per patient \\
\hline & $\begin{array}{l}\text { Coronary Angioplasty Diagnostic Strategy } \\
\text { Including Therapy [64] }\end{array}$ & China & $3,568.00$ & Per patient & $\begin{array}{l}\text { US } \\
(2007)\end{array}$ & $\$ 5,053.37$ & Per patient \\
\hline & $\begin{array}{l}\text { Computed Tomography Angiography and } \\
\text { Coronary Angioplasty Diagnostic Strategy [64] }\end{array}$ & China & $2,971.00$ & Per patient & $\begin{array}{l}\text { US } \\
(2007)\end{array}$ & $\$ 4,207.84$ & Per patient \\
\hline & Inpatient Visit (All direct medical costs) [33] & Argentina & $4,245.39$ & Per patient & $\begin{array}{l}\text { US } \\
(2007)\end{array}$ & $\$ 4,460.54$ & Per patient \\
\hline & Inpatient Visit (All direct medical costs) [41] & China & $11,008.20$ & $\begin{array}{l}\text { Per } \\
\text { inpatient } \\
\text { stay }\end{array}$ & $\begin{array}{l}\text { China } \\
\text { (2003) }\end{array}$ & $\$ 2,304.00$ & $\begin{array}{l}\text { Per } \\
\text { inpatient } \\
\text { stay }\end{array}$ \\
\hline & Inpatient Visit (All direct medical costs) [68] & Vietnam & $31,400,000.00$ & $\begin{array}{l}\text { Per } \\
\text { inpatient } \\
\text { stay }\end{array}$ & $\begin{array}{l}\text { Vietnam } \\
\text { (2005) }\end{array}$ & $\$ 3,257.15$ & $\begin{array}{l}\text { Per } \\
\text { inpatient } \\
\text { stay }\end{array}$ \\
\hline & $\begin{array}{l}\text { Average Inpatient Treatment (All direct } \\
\text { medical costs) [32] }\end{array}$ & $\begin{array}{l}\text { South } \\
\text { Africa }\end{array}$ & $5,636.00$ & Per patient & $\begin{array}{l}\text { US } \\
(2001)\end{array}$ & $\$ 11,093.74$ & Per patient \\
\hline & $\begin{array}{l}\text { Additional cost if a CABG or valve } \\
\text { replacement surgery results in a hospital- } \\
\text { acquired infection [66] }\end{array}$ & India & $14,818.00$ & Per patient & $\begin{array}{l}\text { US } \\
(2006)\end{array}$ & $\$ 21,395.72$ & Per patient \\
\hline & Post CHD with CABG (1st year) [32] & $\begin{array}{l}\text { South } \\
\text { Africa }\end{array}$ & $1,300.00$ & Per year & $\begin{array}{l}\text { US } \\
(2001)\end{array}$ & $\$ 2,558.88$ & $\begin{array}{l}\text { Per patient } \\
\text { per year }\end{array}$ \\
\hline & Post CHD with CABG (subsequent years) [32] & $\begin{array}{l}\text { South } \\
\text { Africa }\end{array}$ & 600.00 & Per year & $\begin{array}{l}\text { US } \\
(2001)\end{array}$ & $\$ 1,181.02$ & $\begin{array}{l}\text { Per patient } \\
\text { per year }\end{array}$ \\
\hline & Post CHD without CABG (1st year) [32] & $\begin{array}{l}\text { South } \\
\text { Africa }\end{array}$ & $1,500.00$ & Per year & $\begin{array}{l}\text { US } \\
(2001)\end{array}$ & $\$ 2,952.56$ & $\begin{array}{l}\text { Per patient } \\
\text { per year }\end{array}$ \\
\hline & $\begin{array}{l}\text { Post CHD without CABG (subsequent years) } \\
\text { [32] }\end{array}$ & $\begin{array}{l}\text { South } \\
\text { Africa }\end{array}$ & 840.00 & Per year & $\begin{array}{l}\text { US } \\
(2001)\end{array}$ & $\$ 1,653.43$ & $\begin{array}{l}\text { Per patient } \\
\text { per year }\end{array}$ \\
\hline \multicolumn{8}{|l|}{ Stroke } \\
\hline \multirow[t]{4}{*}{$\begin{array}{l}\text { Hemorrhagic } \\
\text { Stroke }\end{array}$} & $\begin{array}{l}\text { Inpatient Visit for Stroke Care (Average } 11.8 \\
\text { Days LOS) [17] }\end{array}$ & Turkey & $1,348.00$ & Per patient & $\begin{array}{l}\text { US } \\
(2007)\end{array}$ & $\$ 1,444.46$ & Per patient \\
\hline & $\begin{array}{l}\text { Inpatient Visit for Stroke Care (Average } 12 \\
\text { Days LOS) [18] }\end{array}$ & Brazil & $1,831.00$ & Per patient & $\begin{array}{l}\text { US } \\
(2007)\end{array}$ & $\$ 2,388.33$ & Per patient \\
\hline & $\begin{array}{l}\text { Tertiary Treatment and Follow-Up - Coiling } \\
\text { (6 months) [69] }\end{array}$ & Pakistan & $304,800.00$ & Per patient & $\begin{array}{l}\text { Pakistan } \\
(2007)\end{array}$ & $\$ 6,236.79$ & Per patient \\
\hline & $\begin{array}{l}\text { Tertiary Treatment and Follow-Up - } \\
\text { Endovascular clipping (6 months) [69] }\end{array}$ & Pakistan & $187,620.00$ & Per patient & $\begin{array}{l}\text { Pakistan } \\
\text { (2007) }\end{array}$ & $\$ 3,839.06$ & Per patient \\
\hline $\begin{array}{l}\text { Ischemic } \\
\text { Stroke }\end{array}$ & $\begin{array}{l}\text { Inpatient Visit for Stroke Care (Average } 10.4 \\
\text { Days LOS) [17] }\end{array}$ & Turkey & 956.00 & Per patient & $\begin{array}{l}\text { US } \\
(2007)\end{array}$ & $\$ 1,024.41$ & Per patient \\
\hline
\end{tabular}


Table 2 Unit cost data for the treatment of cardiovascular disease and related condition (USD 2012) (Continued)

\begin{tabular}{|c|c|c|c|c|c|c|c|}
\hline & $\begin{array}{l}\text { Inpatient Visit for Stroke Care (Average } 13.3 \\
\text { Days LOS) [18] }\end{array}$ & Brazil & $1,645.00$ & Per patient & $\begin{array}{l}\text { US } \\
(2007)\end{array}$ & $\$ 2,145.71$ & Per patient \\
\hline & $\begin{array}{l}\text { Inpatient Visit for Stroke Care (Average } 18.5 \\
\text { Days LOS) [70] }\end{array}$ & China & 67.00 & $\begin{array}{l}\text { Per patient } \\
\text { per day }\end{array}$ & $\begin{array}{l}\text { US } \\
(2010)\end{array}$ & $\$ 77.76$ & Per patient \\
\hline & $\begin{array}{l}\text { Inpatient Visit for Stroke Care (Average } 18.5 \\
\text { Days LOS) [70] }\end{array}$ & China & 983.00 & Per patient & $\begin{array}{l}\text { US } \\
(2010)\end{array}$ & $\$ 1,140.84$ & Per patient \\
\hline & $\begin{array}{l}\text { Diagnostics and preventative care for } \\
\text { high-risk patients at a tertiary hospital [71] }\end{array}$ & China & 435.40 & $\begin{array}{l}\text { Per patient } \\
\text { per year }\end{array}$ & $\begin{array}{l}\text { US } \\
(2010)\end{array}$ & $\$ 505.31$ & $\begin{array}{l}\text { Per patient } \\
\text { per year }\end{array}$ \\
\hline \multirow[t]{15}{*}{$\begin{array}{l}\text { Stroke } \\
\text { (Non-Specified) }\end{array}$} & Outpatient Visit [41] & China & 264.80 & $\begin{array}{l}\text { Per } \\
\text { outpatient } \\
\text { visit }\end{array}$ & $\begin{array}{l}\text { China } \\
(2003)\end{array}$ & $\$ 55.42$ & $\begin{array}{l}\text { Per } \\
\text { outpatient } \\
\text { visit }\end{array}$ \\
\hline & $\begin{array}{l}\text { Direct Medical Costs for Managing a Stroke } \\
\text { Patient in Year Following Stroke [72] }\end{array}$ & Nigeria & $62,217.00$ & Per patient & $\begin{array}{l}\text { US } \\
(2012)\end{array}$ & $\$ 62,217.00$ & Per patient \\
\hline & Direct Medical and follow up (6-months) [73] & India & $57,381.00$ & Per patient & $\begin{array}{l}\text { India } \\
(2011)\end{array}$ & $\$ 1,173.80$ & Per patient \\
\hline & $\begin{array}{l}\text { Inpatient Visit for Stroke Care: Average } \\
\text { across all wards (3-5 Days Ave. LOS) [31] }\end{array}$ & Pakistan & $1,179.00$ & Per patient & $\begin{array}{l}\text { US } \\
(2001)\end{array}$ & $\$ 2,160.99$ & Per patient \\
\hline & $\begin{array}{l}\text { Inpatient Visit for Stroke Care: ICU } \\
\text { (3-5 Days LOS) [31] }\end{array}$ & Pakistan & $3,583.50$ & Per patient & $\begin{array}{l}\text { US } \\
(2001)\end{array}$ & $\$ 6,568.20$ & Per patient \\
\hline & $\begin{array}{l}\text { Inpatient Visit for Stroke Care: Private Ward } \\
\text { (3-5 Days LOS) [31] }\end{array}$ & Pakistan & $1,248.00$ & Per patient & $\begin{array}{l}\text { US } \\
(2001)\end{array}$ & $\$ 2,287.46$ & Per patient \\
\hline & $\begin{array}{l}\text { Inpatient Visit for Stroke Care: General Ward } \\
\text { (3-5 Days LOS) [31] }\end{array}$ & Pakistan & $1,010.00$ & Per patient & $\begin{array}{l}\text { US } \\
(2001)\end{array}$ & $\$ 1,851.23$ & Per patient \\
\hline & $\begin{array}{l}\text { Inpatient Visit for Stroke Care } \\
\text { (LOS not stated) [32] }\end{array}$ & $\begin{array}{l}\text { South } \\
\text { Africa }\end{array}$ & $8,633.00$ & Per patient & $\begin{array}{l}\text { US } \\
(2001)\end{array}$ & $\$ 16,992.95$ & Per patient \\
\hline & $\begin{array}{l}\text { Inpatient Visit for Stroke Care } \\
\text { (LOS not stated) [74] }\end{array}$ & Tanzania & $138,000.00$ & Per patient & $\begin{array}{l}\text { Tanzania } \\
(2006)\end{array}$ & $\$ 160.18$ & Per patient \\
\hline & $\begin{array}{l}\text { Inpatient Visit for Stroke Care } \\
\text { (LOS not stated) [33] }\end{array}$ & Argentina & $3,455.48$ & Per patient & $\begin{array}{l}\text { US } \\
(2007)\end{array}$ & $\$ 3,630.60$ & Per patient \\
\hline & $\begin{array}{l}\text { Inpatient Visit for Stroke Care } \\
\text { (Up to } 3 \text { Days LOS) [43] }\end{array}$ & $\begin{array}{l}\text { Congo, } \\
\text { Rep. }\end{array}$ & $158,120.00$ & Per patient & $\begin{array}{l}\text { Congo, } \\
\text { Rep. } \\
(2006)\end{array}$ & $\$ 397.19$ & Per patient \\
\hline & $\begin{array}{l}\text { Inpatient Visit for Stroke Care } \\
\text { (LOS not stated) [41] }\end{array}$ & China & $7,953.10$ & $\begin{array}{l}\text { Per } \\
\text { inpatient } \\
\text { stay }\end{array}$ & $\begin{array}{l}\text { China } \\
(2003)\end{array}$ & $\$ 1,664.57$ & $\begin{array}{l}\text { Per inpatient } \\
\text { stay }\end{array}$ \\
\hline & $\begin{array}{l}\text { Inpatient Visit for Stroke Care: small } \\
\text { hospital (Average } 20 \text { Days LOS) [20] }\end{array}$ & China & $7,119.00$ & $\begin{array}{l}\text { Per } \\
\text { inpatient } \\
\text { stay }\end{array}$ & $\begin{array}{l}\text { China } \\
(2006)\end{array}$ & $\$ 1,388.31$ & $\begin{array}{l}\text { Per inpatient } \\
\text { stay }\end{array}$ \\
\hline & $\begin{array}{l}\text { Inpatient Visit for Stroke Care: tertiary } \\
\text { hospital (Average } 20 \text { Days LOS) [20] }\end{array}$ & China & $12,344.00$ & $\begin{array}{l}\text { Per } \\
\text { inpatient } \\
\text { stay }\end{array}$ & $\begin{array}{l}\text { China } \\
(2006)\end{array}$ & $\$ 2,407.26$ & $\begin{array}{l}\text { Per inpatient } \\
\text { stay }\end{array}$ \\
\hline & $\begin{array}{l}\text { Inpatient Visit for Stroke Care } \\
\text { (Average 6.4 Days LOS) [19] }\end{array}$ & Malaysia & $3,696.40$ & $\begin{array}{l}\text { Per } \\
\text { inpatient } \\
\text { stay }\end{array}$ & $\begin{array}{l}\text { Malaysia } \\
(2005)\end{array}$ & $\$ 1,431.57$ & $\begin{array}{l}\text { Per patient } \\
\text { per admission }\end{array}$ \\
\hline \multicolumn{8}{|l|}{ Heart Failure } \\
\hline & $\begin{array}{l}\text { Inpatient treatment for heart failure due } \\
\text { to systolic or diastolic dysfunction with a } \\
\text { Chagas' cardiomyopathy diagnosis [75] }\end{array}$ & Brazil & 467.00 & Per day & $\begin{array}{l}\text { Brazil } \\
(2006)\end{array}$ & $\$ 324.23$ & Per day \\
\hline & Outpatient Treatment [76] & Brazil & 14.40 & $\begin{array}{l}\text { Per } \\
\text { outpatient } \\
\text { visit }\end{array}$ & $\begin{array}{l}\text { Brazil } \\
(2002)\end{array}$ & $\$ 13.61$ & $\begin{array}{l}\text { Per } \\
\text { outpatient } \\
\text { visit }\end{array}$ \\
\hline & Outpatient Treatment plus medications [76] & Brazil & 557.28 & Per year & $\begin{array}{l}\text { Brazil } \\
(2002)\end{array}$ & $\$ 526.79$ & $\begin{array}{l}\text { Per patient } \\
\text { per year }\end{array}$ \\
\hline & $\begin{array}{l}\text { Inpatient treatment for heart failure due to } \\
\text { systolic or diastolic dysfunction with }\end{array}$ & Brazil & 308.00 & Per day & $\begin{array}{l}\text { Brazil } \\
(2006)\end{array}$ & $\$ 213.84$ & Per day \\
\hline
\end{tabular}


Table 2 Unit cost data for the treatment of cardiovascular disease and related condition (USD 2012) (Continued)

\begin{tabular}{|c|c|c|c|c|c|c|}
\hline Inpatient Care (Up to 3 Days LOS) [43] & $\begin{array}{l}\text { Congo, } \\
\text { Rep. }\end{array}$ & $81,900.00$ & Per patient & $\begin{array}{l}\text { Congo, } \\
\text { Rep. } \\
\text { (2006) }\end{array}$ & $\$ 205.73$ & Per patient \\
\hline Inpatient Care (Average 6.5 Days LOS) [76] & Brazil & $4,033.62$ & $\begin{array}{l}\text { Per } \\
\text { inpatient } \\
\text { stay }\end{array}$ & $\begin{array}{l}\text { Brazil } \\
(2002)\end{array}$ & $\$ 3,812.90$ & $\begin{array}{l}\text { Per inpatien } \\
\text { stay }\end{array}$ \\
\hline
\end{tabular}

Neglected Heart Diseases

\begin{tabular}{|c|c|c|c|c|c|c|c|}
\hline $\begin{array}{l}\text { Congenital } \\
\text { heart disease }\end{array}$ & $\begin{array}{l}\text { Operations and treatments, including pre } \\
\text { and post-operative care [67] }\end{array}$ & Brazil & $1,428.05$ & Per patient & $\begin{array}{l}\text { Brazil } \\
(2001)\end{array}$ & $\$ 1,463.95$ & Per patient \\
\hline \multirow[t]{2}{*}{$\begin{array}{l}\text { Rheumatic } \\
\text { Heart Disease }\end{array}$} & $\begin{array}{l}\text { Inpatient Treatment (Average } 7 \text { Days LOS) } \\
\text { [62] }\end{array}$ & $\begin{array}{l}\text { South } \\
\text { Africa }\end{array}$ & $1,597.00$ & Per patient & $\begin{array}{l}\text { US } \\
(2010)\end{array}$ & $\$ 1,580.49$ & Per patient \\
\hline & $\begin{array}{l}\text { Tertiary care (including inpatient care and } \\
\text { surgery) [61] }\end{array}$ & India & $1,547.17$ & Per patient & $\begin{array}{l}\text { India } \\
(2007)\end{array}$ & $\$ 46.35$ & Per patient \\
\hline \multicolumn{8}{|c|}{ ype Two Diabetes Mellitus } \\
\hline \multirow[t]{8}{*}{$\begin{array}{l}\text { Diabetic Foot } \\
\text { Ulcers }\end{array}$} & Total treatment [21] & Pakistan & $2,700.00$ & Per patient & $\begin{array}{l}\text { Pakistan } \\
\text { (2005) }\end{array}$ & $64.15-\$ 1165.65$ & Per patient \\
\hline & Total Treatment (healed) [22] & China & $1,673.00$ & Per patient & $\begin{array}{l}\$ 1 n t \\
(2010)\end{array}$ & $\$ 585.60$ & Per patient \\
\hline & Total Treatment (trans-tibial amputation) [22] & China & $21,372.00$ & Per patient & $\begin{array}{l}\$ \ln t \\
(2010)\end{array}$ & $\$ 7,480.87$ & Per patient \\
\hline & Total Treatment (healed) [22] & India & $1,192.00$ & Per patient & $\begin{array}{l}\$ \operatorname{lnt} \\
(2010)\end{array}$ & $\$ 85.51$ & Per patient \\
\hline & Total Treatment (trans-tibial amputation) [22] & India & $19,599.00$ & Per patient & $\begin{array}{l}\$ 1 \mathrm{nt} \\
(2010)\end{array}$ & $\$ 1,405.97$ & Per patient \\
\hline & Total Treatment (healed) [22] & Tanzania & 102.00 & Per patient & $\begin{array}{l}\$ \operatorname{lnt} \\
(2010)\end{array}$ & $\$ 0.24$ & Per patient \\
\hline & Total Treatment (trans-tibial amputation) [22] & Tanzania & $3,060.00$ & Per patient & $\begin{array}{l}\text { \$lnt } \\
(2010)\end{array}$ & $\$ 7.30$ & Per patient \\
\hline & Total Treatment [23] & Nigeria & $93,256.70$ & Per patient & $\begin{array}{l}\text { Nigeria } \\
(2003)\end{array}$ & $\$ 1,618.55$ & Per patient \\
\hline \multirow[t]{5}{*}{ Retinopathy } & $\begin{array}{l}\text { Retinopathy screening in a primary care } \\
\text { setting [24] }\end{array}$ & $\begin{array}{l}\text { South } \\
\text { Africa }\end{array}$ & 22.00 & Per patient & $\begin{array}{l}\text { US } \\
(2007)\end{array}$ & $\$ 26.10$ & Per patient \\
\hline & $\begin{array}{l}\text { Laser treatment in retinopathy confirmed } \\
\text { cases [24] }\end{array}$ & $\begin{array}{l}\text { South } \\
\text { Africa }\end{array}$ & 144.00 & Per patient & $\begin{array}{l}\text { US } \\
(2007)\end{array}$ & $\$ 170.85$ & Per patient \\
\hline & $\begin{array}{l}\text { Screening Retinal examination at a hospital } \\
\text { [25] }\end{array}$ & India & 5.84 & Per patient & $\begin{array}{l}\text { US } \\
(2009)\end{array}$ & $\$ 7.05$ & Per patient \\
\hline & $\begin{array}{l}\text { Screening single laser photocoagulation } \\
\text { treatment at a hospital [25] }\end{array}$ & India & 7.51 & Per patient & $\begin{array}{l}\text { US } \\
(2009)\end{array}$ & $\$ 9.07$ & Per patient \\
\hline & $\begin{array}{l}\text { Screening Using Telescreening in rural areas } \\
\text { [25] }\end{array}$ & India & 7.36 & Per patient & $\begin{array}{l}\text { US } \\
(2009)\end{array}$ & $\$ 8.88$ & Per patient \\
\hline \multicolumn{8}{|c|}{ nd-Stage Renal Disease } \\
\hline & Dialysis [29] & China & $56,584.00$ & Per year & $\begin{array}{l}\text { US } \\
(2004)\end{array}$ & $\$ 94,356.62$ & $\begin{array}{l}\text { Per patient } \\
\text { per year }\end{array}$ \\
\hline & Dialysis [29] & Malaysia & $19,054.00$ & Per year & $\begin{array}{l}\text { US } \\
(2004)\end{array}$ & $\$ 28,871.91$ & $\begin{array}{l}\text { Per patient } \\
\text { per year }\end{array}$ \\
\hline & Dialysis [29] & Thailand & $31,651.00$ & Per year & $\begin{array}{l}\text { US } \\
(2004)\end{array}$ & $\$ 52,903.20$ & $\begin{array}{l}\text { Per patient } \\
\text { per year }\end{array}$ \\
\hline & 1 session of hemodialysis in a hospital [27] & Jordan & 72.00 & Per session & $\begin{array}{l}\text { US } \\
(2010)\end{array}$ & $\$ 78.76$ & Per session \\
\hline & $\begin{array}{l}\text { Hemodialysis maintenance session in a } \\
\text { hospital [26] }\end{array}$ & $\begin{array}{l}\text { Iran, } \\
\text { Islamic } \\
\text { Rep. }\end{array}$ & 52.60 & Per session & $\begin{array}{l}\text { US } \\
(2007)\end{array}$ & $\$ 96.66$ & Per session \\
\hline & Hemodialysis in a hospital [28] & Sudan & $15,747.68$ & $\begin{array}{l}\text { Per patient } \\
\text { per year }\end{array}$ & $\begin{array}{l}\text { Sudan } \\
\text { (2009) }\end{array}$ & $\$ 8,374.01$ & $\begin{array}{l}\text { Per patient } \\
\text { per year }\end{array}$ \\
\hline
\end{tabular}


Table 2 Unit cost data for the treatment of cardiovascular disease and related condition (USD 2012) (Continued)

\begin{tabular}{|c|c|c|c|c|c|c|}
\hline Hemodialysis in a hospital [28] & Sudan & 146.58 & $\begin{array}{l}\text { Per patient } \\
\text { per session }\end{array}$ & $\begin{array}{l}\text { Sudan } \\
(2009)\end{array}$ & $\$ 77.95$ & $\begin{array}{l}\text { Per patient } \\
\text { per session }\end{array}$ \\
\hline Renal Transplant (first year expenses) [29] & China & $54,886.00$ & Per year & $\begin{array}{l}\text { US } \\
(2004)\end{array}$ & $\$ 91,525.12$ & $\begin{array}{l}\text { Per patient } \\
\text { per year }\end{array}$ \\
\hline $\begin{array}{l}\text { Maintenance post index year of transplant } \\
\text { patient [29] }\end{array}$ & China & $27,259.00$ & Per year & $\begin{array}{l}\text { US } \\
(2004)\end{array}$ & $\$ 45,455.73$ & $\begin{array}{l}\text { Per patient } \\
\text { per year }\end{array}$ \\
\hline Renal Transplant (first year expenses) [29] & Malaysia & $70,022.00$ & Per year & $\begin{array}{l}\text { US } \\
(2004)\end{array}$ & $\$ 106,102.06$ & $\begin{array}{l}\text { Per patient } \\
\text { per year }\end{array}$ \\
\hline $\begin{array}{l}\text { Maintenance post index year of transplant } \\
\text { patient [29] }\end{array}$ & Malaysia & $14,111.00$ & Per year & $\begin{array}{l}\text { US } \\
(2004)\end{array}$ & $\$ 21,381.94$ & $\begin{array}{l}\text { Per patient } \\
\text { per year }\end{array}$ \\
\hline Renal Transplant (first year expenses) [29] & Thailand & $45,953.00$ & Per year & $\begin{array}{l}\text { US } \\
(2004)\end{array}$ & $\$ 76,808.34$ & $\begin{array}{l}\text { Per patient } \\
\text { per year }\end{array}$ \\
\hline $\begin{array}{l}\text { Maintenance post index year of transplant } \\
\text { patient [29] }\end{array}$ & Thailand & $19,349.00$ & Per year & $\begin{array}{l}\text { US } \\
(2004)\end{array}$ & $\$ 32,340.97$ & $\begin{array}{l}\text { Per patient } \\
\text { per year }\end{array}$ \\
\hline $\begin{array}{l}\text { Kidney transplantation, including operation } \\
\text { and following year [28] }\end{array}$ & Sudan & $34,097.85$ & Per patient & $\begin{array}{l}\text { Sudan } \\
(2009)\end{array}$ & $\$ 18,131.93$ & Per patient \\
\hline Kidney transplantation, after the first year [28] & Sudan & $24,499.00$ & Per patient & $\begin{array}{l}\text { Sudan } \\
\text { (2009) }\end{array}$ & $\$ 13,027.63$ & Per patient \\
\hline Transplantation Procedure [30] & $\begin{array}{l}\text { Iran, } \\
\text { Islamic } \\
\text { Rep. }\end{array}$ & $2,048.00$ & Per patient & $\begin{array}{l}\text { US } \\
(2005)\end{array}$ & $\$ 4,769.20$ & Per patient \\
\hline $\begin{array}{l}\text { Total cost (Transplant procedure, } 1 \text { year } \\
\text { immunosuppression, donor costs) [30] }\end{array}$ & $\begin{array}{l}\text { Iran, } \\
\text { Islamic } \\
\text { Rep. }\end{array}$ & $9,224.00$ & Per patient & $\begin{array}{l}\text { US } \\
(2005)\end{array}$ & $\$ 21,480.05$ & Per patient \\
\hline
\end{tabular}

Definitions: Per capita costs were divided by the population of the study country in 2012 as defined by the World Bank. Primary prevention defined as actions to reduce the probability of initial occurrence of disease. Secondary prevention defined as actions following the occurrence of disease to prevent either the recurrence of the same event or to reduce the risk of a different but related event

Abbreviations: CABG Coronary Artery Bypass Grafting, CHD Coronary Heart Disease, LOS Length of Stay, ICU Intensive Care Unit

ran from $\$ 5,000$ to $\$ 21,000$ in the Sudan and Iran, respectively $[28,30]$.

Other types of CVD, such as heart failure, Chagas cardiomyopathy (CC), congenital heart disease, and rheumatic heart disease, are important contributors to the burden of NCDs in LMICs. However, we identified only a handful of studies on the cost of treating these conditions (Table 2).

\section{Discussion}

Treating CVD and its risk conditions is complex, due in part to the interrelationship between hypertension, diabetes, and ischemic heart disease, and the fact that multiple shared risk factors affect CVD health outcomes. The clinical heterogeneity of CVD can make treatment costs for a single condition much more variable than in the case of infectious diseases or some other chronic diseases; these factors also affect the mean and distribution of costs within and across similar conditions. For instance, CVD encompasses different types of heart and related diseases, such as hypertension, stroke, and heart failure, with different levels of severity, and associated care and management. There are numerous clinical protocols for treating complicated conditions, and treatment includes different combinations of drugs, diagnostics and imaging technologies, surgery and different requirements for inpatient care and follow-up visits, making comparisons between studies all but impossible. Additionally, there are large variations in clinical characteristics, capabilities, and practices among and within countries; it is possible to have a wide distribution of costs even within a hospital if they offer various levels of care, such as general, specialty, and intensive care wards [31].

Another factor affecting the variation in costs was differences in methodology used across studies. There was a lack of standard reporting across the studies, most notably failure to describe cost ingredients. When studies disaggregated costs, there were not clear and common categories for input or activity cost categories. In addition, for disaggregated input costs, it was unclear when activities included personnel in the costs. Many studies provided costs for imaging, diagnostic or therapeutic services, without indicating whether personnel costs were included. Surgery was a common category, but descriptions of what resources comprised surgery or surgical procedures were absent from the majority of the studies. Cost data was most informative when disaggregated into categories and inputs, allowing the reader to better understand heterogeneity and make more useful comparisons. We also noticed a lack of clinical protocol reporting in publications. Reporting on clinical protocols 
underlying the CVD interventions may help readers evaluate the comparability and transferability of costs estimates across studies, depending on the study objective.

Trends in the data emerged despite these limitations. It is clear that there is growing interest and concern about the prevalence and cost of treating cardiovascular and risk conditions at every national income level. BRICS countries and a few other populous Asian countries produced about three-quarters of the literature we identified, laying a foundation of evidence for the costs of treating CVRDs in LMICs. The current evidence confirms that is expensive to provide treatment for complicated or advanced cardiovascular conditions, with inpatient treatment for CVD easily costing upwards of $\$ 10,000$ per patient (Fig. 4). Looking at the distribution of costs by condition, managing risk conditions is generally much less expensive than treating acute CVD conditions (Fig. 5).

Our review identified critical gaps in information and demonstrated the substantial heterogeneity in treatment costs for CVRD across the available data for LMIC countries. The most obvious gap was the need for more consistent methodology, with clear presentation of data on cost ingredients and drivers. Cost data are used to inform resource allocation and to improve efficiency; health policy makers thus need to understand the underlying causes of health expenditures in order to make informed decisions. We identified no longitudinal studies in LMICs, however several modeled studies looked at health costs over a longer period of time. We anticipate that longitudinal studies would be particularly revealing regarding costs of chronic conditions, such as hypertension and diabetes, which require lifelong management. In the absence of longitudinal studies, health decisionmakers are currently allocating CVD resources with an incomplete picture of future costs.

We found very few cost analyses of CVD conditions conducted in low-income countries. While these countries are likely the most heavily resource-constrained, they are also likely the least prepared to prevent and treat CVD conditions. We found no data on the cost of diabetes care in low-income countries. Middle-income countries, particularly upper-middle income countries such as Brazil, China, and South Africa, have produced research or publications regarding technologically complex interventions, revealing relatively higher health system capacity. As the double-burden of infectious and chronic conditions increases, and policy makers in lowincome countries expand health resources for chronic conditions, published studies from other LMICs can provide economic evidence on CVD interventions.

Because our analysis focused on the unit costs of clinical interventions, we did not include many of the WHO Best Buys for NCDs such as tobacco taxation and regulation, population salt regulation, or health and physical activity promotion. Population based prevention measures typically cost pennies per person, but the total cost to the government will depend on the population size. It was beyond the scope of this paper to compare the total costs of population based interventions with clinical prevention and treatment costs. The evidence in this review strongly suggests

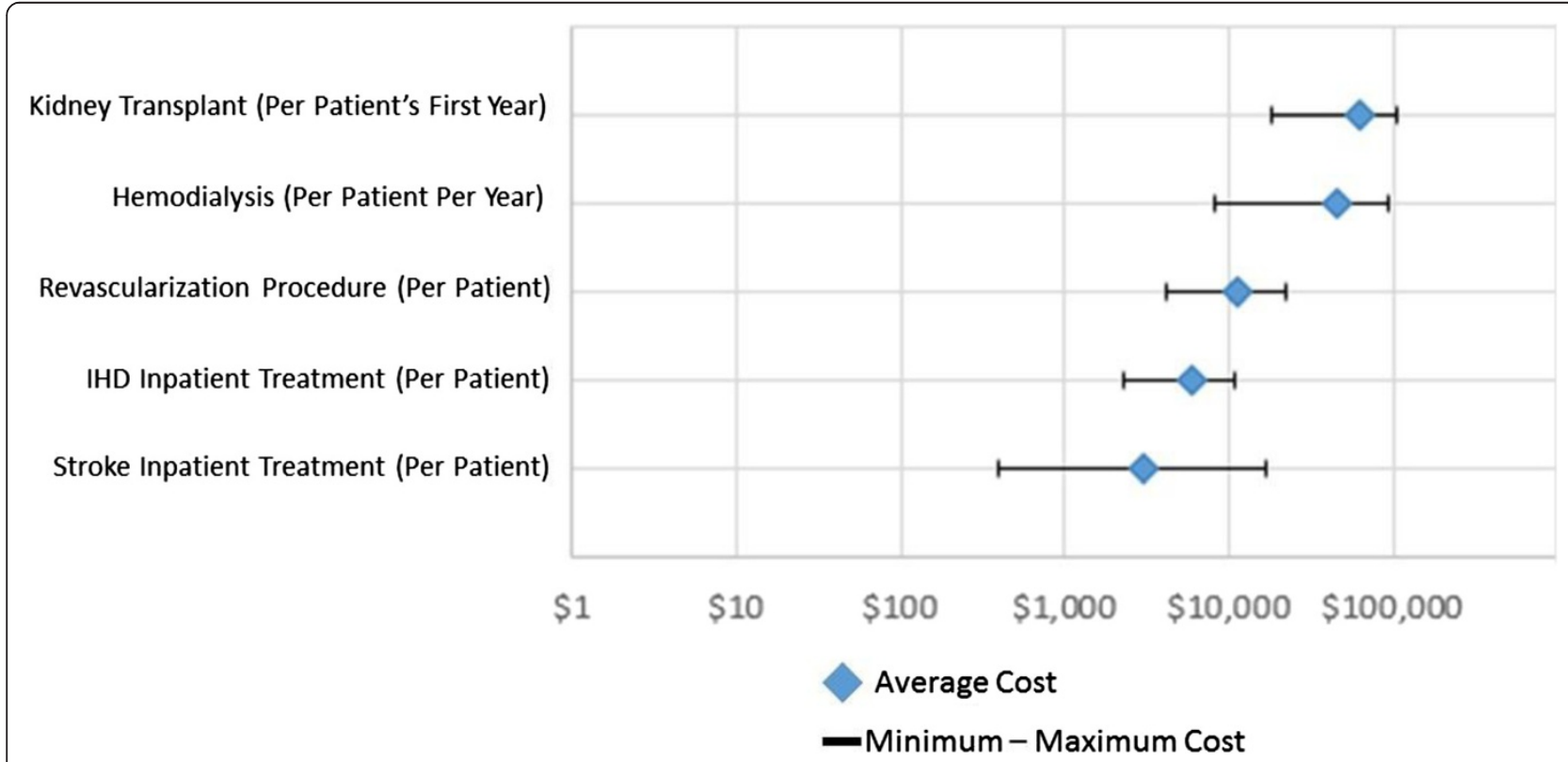

Fig. 4 Hospital Costs by Condition (USD 2012). Showing the average, minimum, and maximum costs of inpatient treatment for various cardiovascular and chronic kidney disease conditions. Source: extracted cost data for this review 


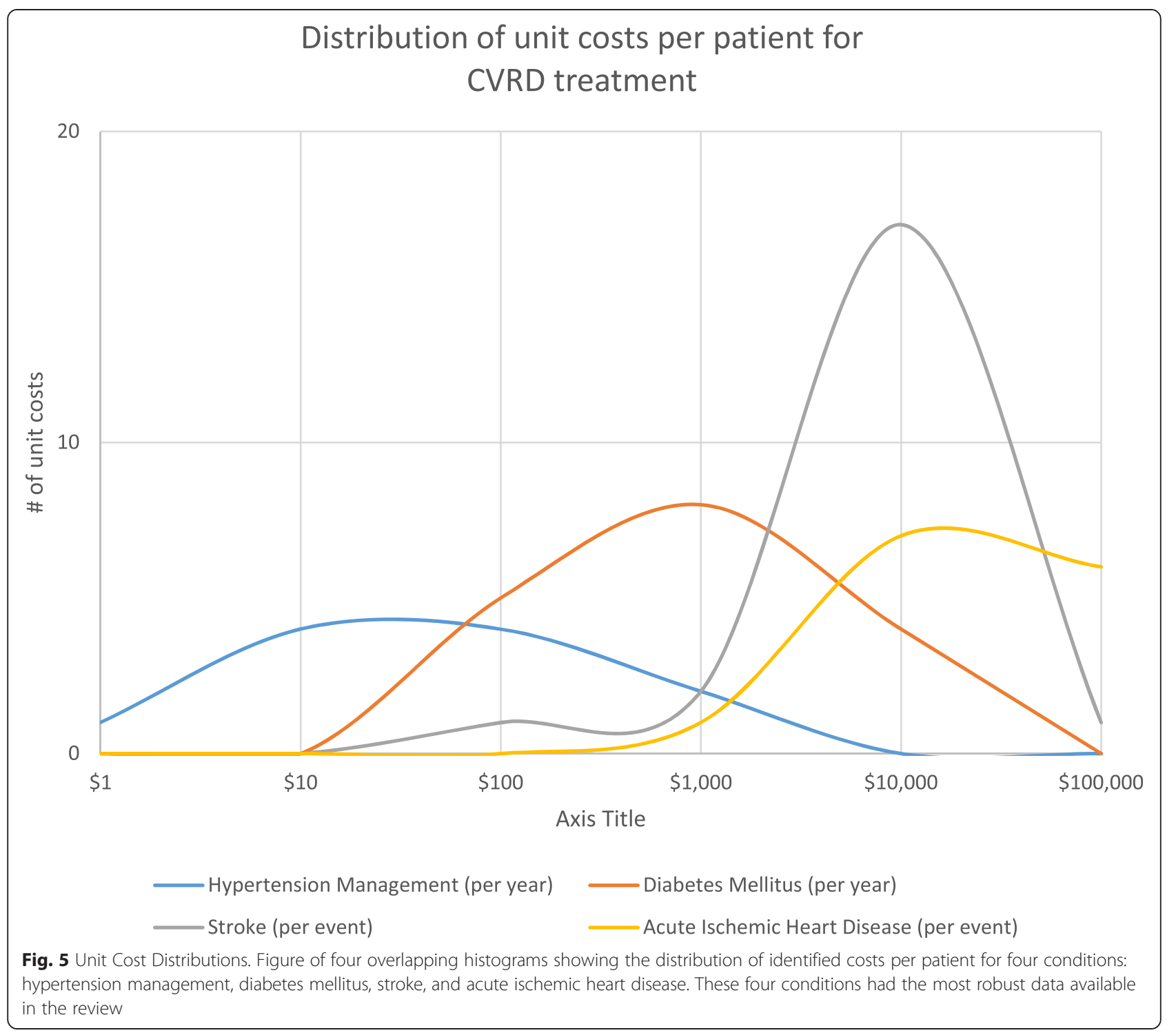

that costs quickly escalate with advanced CVRD treatment; future investment in population prevention of CVRDs could reduce the need for these expensive clinical interventions.

We also excluded studies presenting the cost of implementing guidelines for initiating prevention strategies of CVRDs based on absolute risk [32, 33]. While prevention is extremely important for averting premature death and the high costs of surgery and postoperative care, setting risk guideline thresholds is less associated with changes in unit costs than with changes in long-term cost-effectiveness and total budget impact. The economic evaluations we identified regarding risk guidelines ultimately fell outside of our purview due to their health outcome denominators.

\section{Conclusion}

The evidence for CVRD treatment and prevention costs in LMICs is limited, particularly in low-income countries. We recommend conducting economic studies alongside efforts to scale up treatment and preventions on CVRD in these settings. Initially standard methods of care or clinical protocol could be used to inform costs of CRVD treatment and prevention in order to estimate costs and their key drivers are across settings. There is also a need to estimate the actual resource use and costs in the provision of care, as such information can be used to improve efficiency and budgeting over time. In addition to following recommended costing analysis protocols [15], future research on the costs of CVD prevention and care 
should disaggregate the cost inputs, especially long term costs, since most individuals with CVRD experience worsening disease severity and more sequelae over time.

The burden of CVRD in LMIC is already substantial and will continue to increase in the absence of concerted prevention efforts, which could contain costs by reducing future spending on CVD. At the same time, the prevalence of CVD is already very high in regions such as Latin America and South and East Asia. Hence, efforts to allocate NCD resources equitably will inevitably include a balance of both prevention and advanced treatments for existing cases of CVD. Ongoing efforts to understand the cost of delivering CVRD care in these regions will be critical to achieving universal health coverage and improving overall population health.

\section{Additional files}

Additional file 1: Full search report. (DOCX $21 \mathrm{~kb}$ )

\begin{abstract}
Abbreviations
CKD: Chronic Kidney Disease; CC: chagas cardiomyopathy;

CVD: Cardiovascular disease; CVRD: Cardiovascular Disease and Risk Conditions; DALY: Disability-adjusted life-year; ESRD: End-stage renal disease; LYS: Life years saved; LCU: Local currency unit; LMIC: Low- and middleincome countries; NCD: Non-communicable disease; QALY: Quality-adjusted life-year; T2DM: Type two diabetes mellitus; WHO: World Health Organization.
\end{abstract}

\section{Competing interests}

The authors declare that they have no competing interests.

\section{Authors' contributions}

JG developed and carried out the search strategy. ZO participated in screening the literature and extracting data. DW contributed to conceptualizing the paper and revising drafts of the manuscript. $\mathrm{CL}$ contributed to conceptualizing the paper, analyzing the data, drafting and revising the manuscript. EB participated in screening the literature, extracting data, analyzing the data, and drafting and revising the manuscript. RN contributed to organizing the paper and revising drafts of the manuscript. All authors read and approved the final manuscript.

\section{Acknowledgements}

We thank the Bill \& Melinda Gates Foundation for generous funding through the Disease Control Priorities Network grant to the University of Washington.

\section{Author details}

'Disease Control Priorities Network, Department of Global Health, University of Washington, 325 Ninth Avenue, Box 259931, Seattle, WA 98104, USA. ${ }^{2}$ Department of Medicine, University of Washington, 325 Ninth Ave, Box 359780, Seattle, WA 98104, USA. ${ }^{3}$ School of Public Health, University of California Berkeley, 50 University Hall, \#7360, Berkeley, CA 94720-7360, USA. ${ }^{4}$ PATH, 2201 Westlake Ave \#200, Seattle, WA 98121, USA.

Received: 7 January 2015 Accepted: 23 November 2015 Published online: 26 November 2015

\section{References}

1. Murray CJ, Vos T, Lozano R, Naghavi M, Flaxman AD, Michaud C, et al, Disability-adjusted life years (DALYs) for 291 diseases and injuries in 21 regions, 1990-2010: a systematic analysis for the Global Burden of Disease Study 2010. Lancet. 2012;380(9859):2197-223.

2. Lim S, Vos T, Flaxman A, Danaei G, Shibuya K, Adair-Rohani H, et al. A comparative risk assessment of burden of disease and injury attributable to
67 risk factors and risk factor clusters in 21 regions, 1990-2010: a systematic analysis for the Global Burden of Disease Study 2010. Lancet. 2012; 380(9859):2224-60.

3. Organization WH. Preventing chronic diseases: a vital investment: World Health Organization. 2005.

4. Yusuf S, Rangarajan S, Teo K, Islam S, Li W, Liu L, et al. Cardiovascular Risk and Events in 17 Low-, Middle-, and High-Income Countries. N Engl J Med. 2014;371(9):818-27.

5. Jamison DT, Summers LH, Alleyne G, Arrow KJ, Berkley S, Binagwaho A, et al. Global health 2035: a world converging within a generation. Lancet. 2013;382(9908):1898-955.

6. Kankeu HT, Saksena P, Xu K, Evans DB. The financial burden from noncommunicable diseases in low- and middle-income countries: a literature review. Health Res Policy Syst 2013;11(31).

7. Norheim OF, Jha P, Admasu K, Godal T, Hum RJ, Kruk ME, et al. Avoiding $40 \%$ of the premature deaths in each country, 2010-30: review of national mortality trends to help quantify the UN Sustainable Development Goal for health. Lancet. 2014.

8. World Heart Federation Champion Advocates Program [cited 2014 August 12]. Available from: http://www.championadvocates.org/en

9. Suhrcke M, Boluarte TA, Niessen L. A systematic review of economic evaluations of interventions to tackle cardiovascular disease in low- and middle-income countries. BMC Public Health. 2012;12:2

10. Asaria P, Fortunato L, Fecht D, Tzoulaki I, Abellan JJ, Hambly P, et al. Trends and inequalities in cardiovascular disease mortality across 7932 English electoral wards, 1982-2006: Bayesian spatial analysis. Int J Epidemiol. 2012;41(6):1737-49.

11. Jamison DT, Breman JG, Measham AR, Alleyne G, Claeson M, Evans DB, et al. Disease control priorities in developing countries: World Bank Publications. 2006.

12. Samb B, Desai N, Nishtar S, Mendis S, Bekedam H, Wright A, et al. Prevention and management of chronic disease: a litmus test for healthsystems strengthening in low-income and middle-income countries. Lancet. 2010;376(9754):1785-97.

13. Alam K, Mahal A. Economic impacts of health shocks on households in low and middle income countries: a review of the literature. Glob Health. 2014;10(1):21.

14. Moher D, Liberati A, Tetzlaff J, Altman DG. Preferred reporting items for systematic reviews and meta-analyses: the PRISMA statement. Ann Intern Med. 2009;151(4):264-9.

15. Drummond M, Jefferson T. Guidelines for authors and peer reviewers of economic submissions to the BMJ. The BMJ Economic Evaluation Working Party. BMJ. Br Med J. 1996;313(7052):275.

16. Mogyorosy Z, Smith P. The main methodological issues in costing health care services: a literature review. 2005

17. Asil T, Celik Y, Sut N, Celik AD, Balci K, Yilmaz A, et al. Cost of acute ischemic and hemorrhagic stroke in Turkey. Clin Neurol Neurosurg. 2011;113(2):111-4.

18. Christensen MC, Valiente R, Sampaio Silva G, Lee WC, Dutcher S, Guimaraes Rocha MS, et al. Acute treatment costs of stroke in Brazil. Neuroepidemiology. 2009:32(2):142-9.

19. Nordin N, Aljunid S, Aziz N, Nur AM, Sulong S. Direct Medical Cost of Stroke: Findings from a Tertiary Hospital in Malaysia. Malays J Med Sci. 2012;67(5).

20. Wei JW, Heeley EL, Jan S, Huang Y, Huang Q, Wang JG, et al. Variations and determinants of hospital costs for acute stroke in China. PLoS One. 2010;5(9).

21. Ali SM, Fareed A, Humail SM, Basit A, Ahmedani MY, Fawwad A, et al. The personal cost of diabetic foot disease in the developing world-a study from Pakistan. Diabet Med. 2008;25(10):1231-3.

22. Cavanagh P, Attinger C, Abbas Z, Bal A, Rojas N, Xu Z-R. Cost of treating diabetic foot ulcers in five different countries. Diabetes Metab Res Rev. 2012;28(Suppl 1(dcy, 100883450)):107-11.

23. Ogbera AO, Fasanmade O, Ohwovoriole AE, Adediran O. An assessment of the disease burden of foot ulcers in patients with diabetes mellitus attending a teaching hospital in Lagos, Nigeria. Int J Low Extrem Wounds. 2006;5(4):244-9.

24. Khan T, Bertram MY, Jina R, Mash B, Levitt N, Hofman K. Preventing diabetes blindness: cost effectiveness of a screening programme using digital non-mydriatic fundus photography for diabetic retinopathy in a primary health care setting in South Africa. Diabetes Res Clin Pract. 2013;101(2):170-6

25. Rachapelle S, Legood R, Alavi Y, Lindfield R, Sharma T, Kuper H, et al. The cost-utility of telemedicine to screen for diabetic retinopathy in India. Ophthalmology. 2013;120(3):566-73. 
26. Arefzadeh A, Lessanpezeshki M, Seifi S. The cost of hemodialysis in Iran. Saudi J Kidney Dis Transpl. 2009;20(2):307-11.

27. Al-Shdaifat EA, Manaf MR. The economic burden of hemodialysis in Jordan. Indian J Med Sci. 2013;67(5-6):103-16

28. Elsharif ME, Elsharif EG, Gadour WH. Costs of hemodialysis and kidney transplantation in Sudan: a single center experience. Iran J Kidney Dis. 2010;4(4):282-4.

29. Annemans L, Demarteau N, Hu SS, Lee T, Morad Z, Supaporn T, et al. An Asian Regional Analysis of Cost-Effectiveness of Early Irbesartan Treatment versus Conventional Antihypertensive, Late Amlodipine, and Late Irbesartan Treatments in Patients with Type 2 Diabetes, Hypertension, and Nephropathy. Value Health. 2008;11(3):354-64.

30. Nourbala MH, Einollahi B, Kardavani B, Khoddami-Vishte HR, Assari S, Mahdavi-Mazdeh $\mathrm{M}$, et al. The cost of kidney transplantation in Iran. Transplant Proc. 2007;39(4):927-9.

31. Khealani B, Javed ZF, Syed N, Shafqat S, Wasay M. Cost of Acute Stroke Care at a tertiary care hospital in Karachi, Pakistan. J Pak Med Assoc. 2003;53(11).

32. Gaziano TA, Steyn K, Cohen DJ, Weinstein MC, Opie LH. Cost-effectiveness analysis of hypertension guidelines in South Africa: absolute risk versus blood pressure level. Circulation. 2005;1 12(23):3569-76.

33. Rubinstein A, Colantonio L, Bardach A, Caporale J, Marti SG, Kopitowski K, et al. Estimation of the burden of cardiovascular disease attributable to modifiable risk factors and cost-effectiveness analysis of preventative interventions to reduce this burden in Argentina. BMC Public Health. 2010;10:627.

34. Ilesanmi OS, Ige OK, Adebiyi AO. The managed hypertensive: the costs of blood pressure control in a Nigerian town. Pan Afr Med J. 2012; 12(101517926):96.

35. Robberstad B, Hemed Y, Norheim OF. Cost-effectiveness of medical interventions to prevent cardiovascular disease in a sub-Saharan African country-the case of Tanzania. Cost Eff Resour Alloc. 2007:5:3.

36. Jafar TH, Islam M, Bux R, Poulter N, Hatcher J, Chaturvedi N, et al. Costeffectiveness of community-based strategies for blood pressure control in a low-income developing country: findings from a cluster-randomized, factorial-controlled trial. Circulation. 2011;124(15):1615-25.

37. Bai Y, Zhao Y, Wang G, Wang H, Liu K, Zhao W. Cost-effectiveness of a hypertension control intervention in three community health centers in China. J Prim Care Community Health. 2013;4(3):195-201.

38. Wang $X, L i ~ W, L i X, A n N$, Chen H, Jan $S$, et al. Effects and cost-effectiveness of a guideline-oriented primary healthcare hypertension management program in Beijing, China: results from a 1-year controlled trial. Hypertens Res. 2013:36(4):313-21.

39. Le C, Zhankun $S$, Jun D, Keying Z. The economic burden of hypertension in rural south-west China. Trop Med Int Health. 2012;17(12):1544-51.

40. Pannarunothai S, Kongpan M, Mangklasiri R. Costs-effectiveness of the urban health center in Nakhon Ratchasima: a case study on diabetes and hypertension. J Med Assoc Thai. 2001;84(8):1204-11.

41. Zhao W, Zhai Y, Hu J, Wang J, Yang Z, Kong L, et al. Economic burden of obesity-related chronic diseases in Mainland China. Obes Rev. 2007:9 Suppl 1:62-7.

42. Ngalesoni F, Ruhago G, Robberstad B. Economic cost of primary prevention of cardiovascular diseases in Tanzania. Health policy and planning: Norheim OF; 2014.

43. Gombet TR, Ellenga-Mbolla BF, Ikama MS, Ekoba J, Kimbally-Kaky G. Coût financier de la prise en charge des urgences cardiovasculaires au Centre Hospitalier et Universitaire de Brazzaville. Med Trop. 2009:69:45-7.

44. Sanguantrakul U, Jiamjarasrangsi W, Vimolket T. Efficiency and costeffectiveness of dyslipidemia screening methods among workers in Bangkok. Southeast Asian J Trop Med Public Health. 2010;41(1):215.

45. Wiroj Jiamjarasrangsi M, Wichai Aekplakorn M. Cost and effectiveness of screening methods for abnormal fasting plasma glucose among Thai adults participating in the annual health check-up at King Chulalongkorn Memorial Hospital. J Med Assoc Thai. 2011;94(7):833-41.

46. Zhang Y, Sun J, Pang Z, Gao W, Sintonen $\mathrm{H}$, Kapur A, et al. Evaluation of two screening methods for undiagnosed diabetes in China: an costeffectiveness study. Prim Care Diabetes. 2013;7(4):275-82.

47. Liu X, Li C, Gong H, Cui Z, Fan L, Yu W, et al. An economic evaluation for prevention of diabetes mellitus in a developing country: a modelling study. BMC Public Health. 2013;13(1):1-11.
48. Ramachandran A, Snehalatha C, Yamuna A, Mary S, Ping Z. CostEffectiveness of the Interventions in the Primary Prevention of Diabetes Among Asian Indians. Diabetes Care. 2007;30(10).

49. Wang W, Fu CW, Pan CY, Chen W, Zhan S, Luan R, et al. How Do Type 2 Diabetes Mellitus-Related Chronic Complications Impact Direct Medical Cost in Four Major Cities of Urban China. Value Health. 2009:12(6):923-9.

50. Riewpaiboon A, Pornlertwadee P, Pongsawat K. Diabetes cost model of a hospital in Thailand. Value Health. 2007;10(4):223-30.

51. Abdulganiyu G, Fola T. What is the cost of illness of type II Diabetes Mellitus in a Developing Economy? Int J Pharm Pharm Sci. 2014;6 Suppl 2:927-31.

52. Cobas RA, Ferraz MB, Matheus AS, Tannus LR, Negrato CA, Antonio de Araujo $L$, et al. The cost of type 1 diabetes: a nationwide multicentre study in Brazil. Bull World Health Organ. 2013;91(6):434-40.

53. Akari S, Mateti UV, Kunduru BR. Health-care cost of diabetes in South India: A cost of illness study. J Res Pharm Pract. 2013;2(3):114-7.

54. Tharkar S, Satyavani K, Viswanathan V. Cost of medical care among type 2 diabetic patients with a co-morbid condition-hypertension in India. Diabetes Res Clin Pract. 2009;83(2):263-7.

55. Li H, Chen BK, Shah N, Wang Z, Eggleston KN. Socioeconomic correlates of inpatient spending for patients with type 2 diabetes mellitus in China: evidence from Hangzhou. Exp Clin Endocrinol Diabetes. 2012;120(1):35-44.

56. Chatterjee S, Riewpaiboon A, Piyauthakit P, Riewpaiboon W, Boupaijit K, Panpuwong $\mathrm{N}$, et al. Cost of diabetes and its complications in Thailand: a complete picture of economic burden. Health Soc Care Community. 2011;19(3):289-98.

57. Kumpatla S, Kothandan H, Tharkar S, Viswanathan V. The Costs of Treating Long Term Diabetic Complications in a Developing Country: A Study from India. J Assoc Physicians India. 2013;61.

58. Shrestha N, Lohani S, Angdembe M, Bhattarai K, Bhattarai J. Cost of Diabetes Mellitus Care among Patients attending selected Outpatient Clinics. J Nepal Med Assoc. 2013;52(190):343-8.

59. Bahia LR, Araujo DV, Schaan BD, Dib SA, Negrato CA, Leao MP, et al. The costs of type 2 diabetes mellitus outpatient care in the Brazilian public health system. Value Health. 2011;14(5 Suppl 1):S137-40.

60. Khowaja LA, Khuwaja AK, Cosgrove P. Cost of diabetes care in out-patient clinics of Karachi, Pakistan. BMC Health Serv Res. 2007;7(101088677):189.

61. Soudarssanane MB, Karthigeyan M, Sahai A, Srinivasan S, Rao KS, Balachander J. Rheumatic fever and rheumatic heart disease: primary prevention is the cost effective option. Indian J Pediatr. 2007;74(6):567-70.

62. Irlam J, Mayosi BM, Engel M, Gaziano TA. Primary prevention of acute rheumatic fever and rheumatic heart disease with penicillin in South African children with pharyngitis: a cost-effectiveness analysis. Circ Cardiovasc Qual Outcomes. 2013;6(3):343-51.

63. Reeves BM, Kado J, Brook M. High prevalence of rheumatic heart disease in Fiji detected by echocardiography screening. J Paediatr Child Health. 2011;47(7):473-8.

64. Cheng MM, Lu B, Hu SS, Marelli C, Higashi MK, Patel PA, et al. Optimizing CAD diagnosis in China with CT angiography. Cardiovasc Comput Tomogr. 2009;3(3):153-8.

65. Perikhanyan A. Effectiveness and cost-effectiveness of coronary artery bypass surgery versus drug eluting stents in armenia: a feasibility study. Georgian Med News. 2011;6(195):44-51.

66. Kothari A, Sagar V, Ahluwalia V, Pillai BS, Madan M. Costs associated with hospital-acquired bacteraemia in an Indian hospital: a case-control study. J Hosp Infect. 2009;71(2):143-8.

67. Fernandes A, Mansur AJ, Canêo LF, Lourenço DD, Piccioni MA, Franchi SM, et al. The Reduction in Hospital Stay and Costs in the Care of Patients with Congenital Heart Diseases Undergoing Fast-Track Cardiac Surgery. Arq Bras Cardiol. 2004;83(1):27-34

68. Ross $\mathrm{H}$, Trung DV, Phu VX. The costs of smoking in Vietnam: the case of inpatient care. Tob Control. 2007;16(6):405-9.

69. Zubair Tahir M, Enam SA, Pervez Ali R, Bhatti A, ul Haq T. Cost-effectiveness of clipping vs coiling of intracranial aneurysms after subarachnoid hemorrhage in a developing country-a prospective study. Surg Neurol. 2009;72(4):355-60. discussion 60-1.

70. Ma Y, Liu Y, Fu HM, Wang XM, Wu BH, Wang SX, et al. Evaluation of admission characteristics, hospital length of stay and costs for cerebral infarction in a medium-sized city in China. Eur J Neurol. 2010;17(10):1270-6

71. Zhao JJ, He GQ, Gong SY, He L. Status and costs of primary prevention for ischemic stroke in China. J Clin Neurosci. 2013;20(10):1427-32. 
72. Birabi BN, Oke Kl, Dienye PO, Okafor UC. Cost burden of post stroke condition in Nigeria: a pilot study. Glob J Health Sci. 2012;4(6):17-22.

73. Kwatra G, Kaur P, Toor G, Badyal DK, Kaur R, Singh Y, et al. Cost of stroke from a tertiary center in northwest India. Neurol India. 2013;61(6):627-32.

74. Kabadi GS, Walker R, Donaldson C, Shackley P. The cost of treating stroke in urban and rural Tanzania: A 6-month pilot study. African Journal of Neurological Sciences. 2013;32(2).

75. Abuhab A, Trindade E, Aulicino GB, Fujii S, Bocchi EA, Bacal F. Chagas' cardiomyopathy: the economic burden of an expensive and neglected disease. Int J Cardiol. 2013;168(3):2375-80,

76. Araujo DV, Tavares $L R$, Verissimo R, Ferraz MB, Mesquita E. Cost of Heart Failure in the Unified Health System. Arq Bras Cardiol. 2005;84(5):422-7.

Submit your next manuscript to BioMed Central and we will help you at every step:

- We accept pre-submission inquiries

- Our selector tool helps you to find the most relevant journal

- We provide round the clock customer support

- Convenient online submission

- Thorough peer review

- Inclusion in PubMed and all major indexing services

- Maximum visibility for your research 